\title{
On symmetries of crystals with defects related to a class of solvable groups $\left(S_{2}\right)$
}

\author{
Rachel Nicks ${ }^{1}$ and Gareth P. Parry ${ }^{2}$ \\ School of Mathematical Sciences, \\ University of Nottingham, Nottingham NG7 2RD, UK
}

\begin{abstract}
We find the geometrical symmetries of discrete structures which generalize the perfect lattices of crystallography to account for the existence of continuous distributions of defects.
\end{abstract}

Keywords Crystals · Defects · Lie Groups

Mathematics Subject Classification (2000) 74A20 · 74E25

\section{Introduction}

The study of the geometrical symmetries of perfect lattices is a starting point for the mechanics of solid crystals. Traditionally, one finds the point group symmetries of a given lattice, hypothesizes that a continuum strain energy density has material symmetry group which contains those point group (rotational) symmetries and constructs energy functions invariant under the rotational transformations associated with the classical crystals classes (triclinic, tetragonal, cubic, etc.). In fact, in nonlinear elasticity the strain energy is expressed as a function of Green strain (which is effectively a metric measuring deformations of a continuum), and the connection between the rotational symmetries of the lattice and the corresponding transformations of the Green strain is predicated on an assumption that the basis vectors of the discrete lattice are embedded in deformations of the corresponding continuum. This assumption is the CauchyBorn hypothesis, in essence, and it connects discrete and continuum descriptions of the crystal.

Now crystals do not always deform elastically, e.g., for large enough shear stresses, it is common to see crystal 'slip', when certain parallel planes of atoms slide one with respect to the other. So, it is well recognized that the Cauchy-Born hypothesis does not always hold. In this paper we focus on a continuum model where the basic kinematical variables are three linearly

\footnotetext{
${ }^{1}$ née Sigrist

${ }^{2}$ Corresponding author: G.P. Parry, School of Mathematical Sciences, University of Nottingham, Nottingham NG7 2RD, UK. email: gareth.parry@nottingham.ac.uk
} 
independent vectors (which we call 'lattice vectors') rather than the Green strain tensor. A crystal configuration, then, is a distribution where these three vectors are prescribed at each point of a given region - a 'field' of three linearly independent, smoothly varying, lattice vectors. We do not assume that, if a mapping of the points of the region were to be given, the lattice vector fields would transform as do embedded line elements in the mapping. So we allow that the Cauchy-Born hypothesis may or may not hold, when one compares different crystal configurations. (When two configurations are such that the hypothesis does hold, we say that the lattice vector fields transform elastically).

We also allow that the lattice vector fields do not commute with one another, by way of contrast with the case of perfect crystals, where configurations of interest are fields of vectors which represent elastic deformations of constant fields (constant in the sense that they are unchanged from point to point in $\mathbb{R}^{3}$ ). So, first of all in this paper, we discuss different measures of non-commutativity of vector fields, and give details of the connection between the notions of Lie bracket, Burgers' vectors and dislocation density tensor. Then, we specify the extent to which this work generalizes the continuum (elastic) theory of the mechanics of crystals - it simply amounts to replacing the dependence of strain energy on Green strain by a dependence on point values of the lattice vector fields and dislocation density tensor.

Next, we make a central assumption, that the values of the lattice vector fields and the dislocation density tensor determine a geometrical structure which plays the same role in this general theory as the perfect lattice plays in continuum models of perfect crystals. This assumption lead to the construction described in the next paragraph, which generalizes the way that a perfect lattice is constructed from its basis given that relevant vector fields are commutative.

So far as the dislocation density tensor is concerned (this is denoted $S$, and involves first gradients of the lattice vector fields) we are given only one value, as an argument of the strain energy, and have no information about the gradients of $S$. Therefore, in constructing 'local' crystal configurations which the arguments of the strain energy are meant to represent, we choose to consider distributions of vector fields where $S$ is constant in space, so its spatial gradients are zero, and we call such configurations of lattice vector fields 'uniform'. We show that these uniform configurations have a Lie group structure, i.e., that points in the region represent group elements, and that there is an (associative) binary composition of group elements which is determined by the value of $S$ (it turns out that $S$ is closely related to the structure constants of the Lie group). Note that in the perfect crystal case one obtains in 
this way a configuration where the lattice vector fields are 'translation invariant', and the corresponding Lie group is $\mathbb{R}^{3}$ with addition of points as group operation. In the general case, the Lie group structure requires that the distribution of lattice vector fields has the self-similarity specified by equation (3.7) below. Also, the perfect lattice is obtained from the translation invariant fields by a certain iteration procedure - the generalization of that procedure gives a subgroup of the Lie group, $G$, that is determined by $S$. We discuss these issues in sections 3 and 4, and remark that in classical crystallography there is a further restriction which is employed to construct sets of points meant to model crystal structure, that restriction is to confine attention to discrete sets of points, so that there is a positive minimum separation between pairs of points in the structure. It turns out, according to Auslander, Green and Hahn [6], Thurston [16], that when this restriction (requiring discreteness) is applied to the set of points obtained by the iteration procedure, there is a corresponding restriction on the structure of the Lie group (and similar restrictions on $S$ ). In fact there are just three classes of three dimensional Lie group which have discrete subgroups - two of these are classes of solvable groups, one is a class of nilpotent groups.

Our main task will be to investigate what types of symmetry should be applied to continuum energy functions which model crystals whose structure relates to the uniform lattice vector fields described above, and we will do this by considering the different sets of group elements which generate the corresponding discrete subgroups (just as, in crystallography, one considers the different sets of basis vectors which generate a perfect lattice). In fact, we have dealt with two of the three classes of discrete groups in previous work, Parry and Sigrist [10], Nicks and Parry [11], so here we complete the process by considering just the class of solvable groups that Auslander, Green and Hahn [6] call $S_{2}$.

To proceed, we construct the derived group, denoted $G^{\prime}$, of $G$. Let $(\boldsymbol{g}, \boldsymbol{h}) \equiv \boldsymbol{g}^{-1} \boldsymbol{h}^{-1} \boldsymbol{g} \boldsymbol{h}, \boldsymbol{g}, \boldsymbol{h} \in$ $G$, be the commutator of group elements $\boldsymbol{g}$ and $\boldsymbol{h}$-this plays the same role in the theory that we describe as the Burgers' vectors play in descriptive treatments of defects in crystals modelled as collections of atoms, or discrete sets of points. In Lie theory, the group that is generated by the commutators of group elements and their inverses is called the derived group, or commutator subgroup, of $G$. For the particular nilpotent or solvable three dimensional Lie groups that have discrete subgroups, it is a fact that the elements of $G^{\prime}$ commute with each other, and that binary composition (in $G$ ) reduces to addition in $G^{\prime}$. If we restrict attention, further, to the discrete subgroups of $G$, denoted $\mathcal{D}$, then $\mathcal{D}^{\prime}$ (defined in the obvious way) is a two dimensional lattice, so we can (and do) find the basis vectors of this two dimensional lattice (cf. (6.14)). In different language, $\mathcal{D}^{\prime}$ represents all vectors which can be constructed by composition of Burgers' vectors. 
To find the symmetries of $\mathcal{D}$, which we interpret to mean finding the set of all generators of $\mathcal{D}$, we proceed as follows. Pick three elements $\boldsymbol{g}_{1}, \boldsymbol{g}_{2}, \boldsymbol{g}_{3} \in \mathcal{D}$ and construct the group which consists of all products of $\boldsymbol{g}_{1}, \boldsymbol{g}_{2}, \boldsymbol{g}_{3}$ and their inverses, denoted $\mathcal{G} \equiv g p\left(\boldsymbol{g}_{1}, \boldsymbol{g}_{2}, \boldsymbol{g}_{3}\right)$. In the case that the relevant Lie group $G$ is $\mathbb{R}^{3}$ with addition as binary operation, $\mathcal{G}$ has elements which correspond to the points of a perfect lattice with $\boldsymbol{g}_{1}, \boldsymbol{g}_{2}, \boldsymbol{g}_{3}$ as basis vectors. When the distribution of lattice vector fields is uniform, and the corresponding discrete subgroup $\mathcal{G}$ is generated according to the composition function (binary operation) given by (5.13), we note the following statement of Bachmuth [14], that the general form of an element of $\mathcal{G}$ is given by expression (7.14). This expression is a generalization of the expression for a general element of a lattice as an integer linear combination of basis vectors to the case of discrete subgroups of Lie groups where commutators commute - as such it involves products of the generators and their inverses, but also the 'fundamental' commutators $\left(\boldsymbol{g}_{1}, \boldsymbol{g}_{2}\right),\left(\boldsymbol{g}_{2}, \boldsymbol{g}_{3}\right),\left(\boldsymbol{g}_{3}, \boldsymbol{g}_{1}\right)$ which are generators of $\mathcal{G}^{\prime}$.

It turns out that the conditions on $\boldsymbol{g}_{1}, \boldsymbol{g}_{2}, \boldsymbol{g}_{3}$ such that $\mathcal{G}^{\prime}=\mathcal{D}^{\prime}$ are necessary and sufficient that $\mathcal{G}=\mathcal{D}$. So, since commutators commute and the binary operation in $\mathcal{G}^{\prime}$ is addition, and we know basis vectors for $\mathcal{D}^{\prime}$, the task is reduced to a problem in linear algebra. This enables us to find detailed conditions on a certain representation of $\boldsymbol{g}_{1}, \boldsymbol{g}_{2}, \boldsymbol{g}_{3}$ which guarantee that these group elements generate the discrete subgroup $\mathcal{D}$.

Finally, it becomes clear that there are interesting qualitative differences between the symmetries that we have found, and the symmetries that correspond to changes of basis of perfect lattices. These relate, in the main, to whether or not changes of generators (of $\mathcal{D}$ ) extend to automorphisms of the continuous group $G$ (i.e., extend to elastic deformations, preserving the dislocation density). It appears that such extensions exist for some choices of $S$, not for others. We postpone detailed discussion of issues like this for future work, but remark that it would seem to provide a rigorous geometric classification of discrete symmetries into elastic and inelastic classes, at the least.

\section{Integral curves of vector fields, non-commutativity}

Let $\boldsymbol{\ell}(\cdot)$ be a vector field defined on $\mathbb{R}^{3}$. Let $\boldsymbol{x}\left(t, \boldsymbol{x}_{0}\right)$ be the solution of the following ordinary differential equation:

$$
\frac{d}{d t} \boldsymbol{x}\left(t, \boldsymbol{x}_{0}\right)=\boldsymbol{\ell}\left(\boldsymbol{x}\left(t, \boldsymbol{x}_{0}\right)\right), \quad \boldsymbol{x}\left(0, \boldsymbol{x}_{0}\right)=\boldsymbol{x}_{0} .
$$


Then $\left\{\boldsymbol{x}\left(t, \boldsymbol{x}_{0}\right): t \in \mathbb{R}\right\}$ is the integral curve of the vector field $\boldsymbol{\ell}(\cdot)$, passing through the point $\boldsymbol{x}_{0}$. We say that $\boldsymbol{x}$ is the flow generated by the field $\boldsymbol{\ell}(\cdot)$, and introduce the mapping $\exp (t \boldsymbol{\ell})=$ $\mathbb{R}^{3} \rightarrow \mathbb{R}^{3}$ by

$$
\exp (t \boldsymbol{\ell}) \boldsymbol{x}_{0}=\boldsymbol{x}\left(t, \boldsymbol{x}_{0}\right)
$$

One understands that the symbol $\boldsymbol{\ell}$, in the expression $\exp (t \ell)$, represents the field $\boldsymbol{\ell}(\cdot)$. Then, according to Olver [1], for example,

$$
\begin{aligned}
& \exp (t \boldsymbol{\ell}) \exp (s \boldsymbol{\ell})=\exp (t+s) \boldsymbol{\ell}, \quad t, s \in \mathbb{R}, \\
& \exp (-t \boldsymbol{\ell})=\{\exp (t \boldsymbol{\ell})\}^{-1}, \\
& \frac{d}{d t}[\exp (t \boldsymbol{\ell}) \boldsymbol{x}]=\boldsymbol{\ell}(\exp (t \boldsymbol{\ell}) \boldsymbol{x}) .
\end{aligned}
$$

We focus on the particular element of the flow which maps a point $\boldsymbol{x}_{0} \in \mathbb{R}^{3}$ to $\boldsymbol{x}\left(1, \boldsymbol{x}_{0}\right)$, denoted $\exp (\boldsymbol{\ell})$. We say that $\exp (\boldsymbol{\ell})$ represents an 'iteration' of points $\boldsymbol{x}_{0} \in \mathbb{R}^{3}$ along the vector field $\ell(\cdot)$. Define the point $e^{\ell}$ (as opposed to the mapping $\exp (\ell)$ ) by

$$
e^{\ell}=\exp (\ell) \mathbf{0}
$$

so that $e^{\ell}$ is that point obtained by (a single) iteration of the origin $\mathbf{0} \in \mathbb{R}^{3}$ along the vector field $\ell(\cdot)$. Points $e^{t \ell}$ are defined analogously.

Now let $\boldsymbol{\ell}_{1}(\cdot)$ and $\boldsymbol{\ell}_{2}(\cdot)$ be two different fields defined as $\mathbb{R}^{3}$, and form the quantity

$$
\theta\left(t, \boldsymbol{x}_{0}\right)=\exp \left(-\sqrt{t} \boldsymbol{\ell}_{2}\right) \exp \left(-\sqrt{t} \boldsymbol{\ell}_{1}\right) \exp \left(\sqrt{t} \boldsymbol{\ell}_{2}\right) \exp \left(\sqrt{t} \boldsymbol{\ell}_{1}\right) \boldsymbol{x}_{0}
$$

which corresponds to successive flows along the fields $\boldsymbol{\ell}_{1}(\cdot), \boldsymbol{\ell}_{2}(\cdot),-\boldsymbol{\ell}_{1}(\cdot),-\boldsymbol{\ell}_{2}(\cdot)$. The derivative of this quantity, with respect to $t$, at $t=0$, defines the Lie bracket $\left[\ell_{1}, \ell_{2}\right]$ of the vector fields $\boldsymbol{\ell}_{1}(\cdot)$ and $\boldsymbol{\ell}_{2}(\cdot)$, see Olver [1]. One may calculate that

$$
\left[\ell_{1}, \ell_{2}\right]=\left(\ell_{1} \cdot \nabla\right) \ell_{2}-\left(\ell_{2} \cdot \nabla\right) \ell_{1},
$$

when the right hand side of $(2.6)$ is evaluated at the point $\boldsymbol{x}_{0}$. The fields $\boldsymbol{\ell}_{1}(\cdot)$ and $\boldsymbol{\ell}_{2}(\cdot)$ commute, in the sense that $\theta\left(t, \boldsymbol{x}_{0}\right)$ is zero for all $\boldsymbol{x}_{0} \in \mathbb{R}^{3}$, if and only if $\left[\boldsymbol{\ell}_{1}, \boldsymbol{\ell}_{2}\right]$ is identically zero, so the Lie bracket is a measure of the non-commutativity of the two fields.

We can define a different, but related, measure of non-commutativity as follows. Let $\boldsymbol{\ell}_{1}(\cdot), \boldsymbol{\ell}_{2}(\cdot), \boldsymbol{\ell}_{3}(\cdot)$ be three linearly independent fields on $\mathbb{R}^{3}$, and let $\boldsymbol{d}_{1}(\cdot), \boldsymbol{d}_{2}(\cdot), \boldsymbol{d}_{3}(\cdot)$ be the corresponding dual fields, so

$$
\boldsymbol{\ell}_{i}(\boldsymbol{x}) \cdot \boldsymbol{d}_{j}(\boldsymbol{x})=\delta_{i j}, \quad i, j=1,2,3, \boldsymbol{x} \in \mathbb{R}^{3}
$$


where $\left(\delta_{i j}\right)$ is the Kronecker delta. Also define

$$
n(\boldsymbol{x})=\boldsymbol{d}_{1}(\boldsymbol{x}) \cdot \boldsymbol{d}_{2}(\boldsymbol{x}) \wedge \boldsymbol{d}_{3}(\boldsymbol{x}), \quad \boldsymbol{x} \in \mathbb{R}^{3} .
$$

This gives

$$
\boldsymbol{d}_{1}=n \boldsymbol{\ell}_{2} \wedge \boldsymbol{\ell}_{3} \text {, etc., }
$$

and by considering the cofactors of elements in the matrix whose columns, or rows, are the dual fields $\boldsymbol{d}_{1}(\cdot), \boldsymbol{d}_{2}(\cdot), \boldsymbol{d}_{3}(\cdot)$, one obtains

$$
\varepsilon_{a b c} d_{c i}=\varepsilon_{i j k} n \ell_{a j} \ell_{b k}, \quad \varepsilon_{a b c} d_{i c}=\varepsilon_{i j k} n \ell_{j a} \ell_{k b}
$$

where $\left(\varepsilon_{a b c}\right)$ is the presentation symbol, the summation convention operates on repeated indices, and the components of $\boldsymbol{d}_{i}, \ell_{j}$ are $\left(d_{i p}\right),\left(\ell_{j q}\right), i, j, p, q=1,2,3$, respectively. Let $C$ be a circuit in $\mathbb{R}^{3}$,

$$
C=\{\boldsymbol{x}(t): t \in[0,1], \boldsymbol{x}(1)=\boldsymbol{x}(0)\} .
$$

Construct a related path $P \equiv\{\boldsymbol{y}(t) ; t \in[0,1]\}$ by associating with each 'line element' $d \boldsymbol{x}$ of $C$ a corresponding increment $\boldsymbol{d} \boldsymbol{y}=\left(\boldsymbol{d} \boldsymbol{x} \cdot \boldsymbol{d}_{a}\right) \boldsymbol{e}_{a}$ of $P$, where $\left\{\boldsymbol{e}_{1}, \boldsymbol{e}_{2}, \boldsymbol{e}_{3}\right\}$ is a basis of $\mathbb{R}^{3}$ (note that $\left.\boldsymbol{d} \boldsymbol{x}=\left(\boldsymbol{d} \boldsymbol{x} \cdot \boldsymbol{d}_{a}\right) \boldsymbol{\ell}_{a}\right)$. That is, let $\boldsymbol{y}(t)$ be the solution of the differential equation

$$
\dot{\boldsymbol{y}}=\left(\dot{\boldsymbol{x}} \cdot \boldsymbol{d}_{a}\right) \boldsymbol{e}_{a} .
$$

Then, whilst

$$
\boldsymbol{x}(1)-\boldsymbol{x}(0)=\mathbf{0}=\int_{0}^{1} \dot{\boldsymbol{x}}(t) d t=\int_{0}^{1} \boldsymbol{\ell}_{a}\left(\boldsymbol{d}_{a} \cdot \boldsymbol{d}_{x}\right),
$$

we have

$$
\boldsymbol{y}(1)-\boldsymbol{y}(0)=\int_{0}^{1} \dot{\boldsymbol{y}}(t) d t=e_{a} \oint_{C} \boldsymbol{d}_{a} \cdot \boldsymbol{d}_{x}=e_{a} \int_{\mathcal{S}} \nabla \wedge \boldsymbol{d}_{a} \cdot \boldsymbol{d} \mathcal{S}
$$

when $\mathcal{S}$ is any surface with boundary $C$. So the 'closure failure', $\boldsymbol{y}(1)-\boldsymbol{y}(0)$, of the path $P$ is determined by the fields of Burgers' vectors $\nabla \wedge \boldsymbol{d}_{a}(\cdot), a=1,2,3$. This argument is often described by stating that a circuit $C$ in a defective crystal with increments $\boldsymbol{d} \boldsymbol{x}$ parallel to lattice vector fields (different lattice vector fields at different points) maps to a path $P$, in a perfect crystal lattice, that does not close, generally. (For example, an increment $\boldsymbol{d x}=\alpha \boldsymbol{\ell}_{1}(\boldsymbol{x})$ of $C$ has $\boldsymbol{d} \boldsymbol{x} \cdot \boldsymbol{d}_{1}=\alpha \boldsymbol{d} \boldsymbol{x} \cdot \boldsymbol{d}_{2}=\boldsymbol{d} \boldsymbol{x} \cdot \boldsymbol{d}_{3}=0$ and this maps to an increment $\boldsymbol{d} \boldsymbol{y}=\alpha \boldsymbol{e}_{1}$ of $P$.) So the Burgers' vectors also measure non-commutativity. 
There is a simple relation between the Burgers' vectors and the Lie brackets of the three pairs of fields $\boldsymbol{\ell}_{1}(\cdot), \boldsymbol{\ell}_{2}(\cdot) ; \boldsymbol{\ell}_{2}(\cdot), \boldsymbol{\ell}_{3}(\cdot) ; \boldsymbol{\ell}_{3}(\cdot), \boldsymbol{\ell}_{1}(\cdot)$, which we denote by $\boldsymbol{L}_{3}(\cdot), \boldsymbol{L}_{1}(\cdot), \boldsymbol{L}_{2}(\cdot)$ respectively. To see this, put

$$
\boldsymbol{L}_{a} \equiv \varepsilon_{a c b}\left(\boldsymbol{\ell}_{b} \cdot \nabla\right) \boldsymbol{\ell}_{c} .
$$

Then

$$
\begin{array}{rlrl}
n \boldsymbol{L}_{a} \cdot \boldsymbol{d}_{r} & \equiv n \varepsilon_{a c b}\left(\boldsymbol{\ell}_{b} \cdot \nabla\right) \boldsymbol{\ell}_{c} \cdot \boldsymbol{d}_{r} & \\
& =n \varepsilon_{a c b} \ell_{b j} \ell_{c k, j} d_{r k} & \\
& =n \varepsilon_{a b c} \ell_{b j} \ell_{c k} d_{r k, j}, & & \text { since } \boldsymbol{\ell}_{c} \cdot \boldsymbol{d}_{r}=\delta_{c r}, \\
& =\varepsilon_{j k i} d_{a i} d_{r k, j}, & & \text { via }(2.10)_{2} \\
& \equiv d_{a i}\left(\nabla \wedge \boldsymbol{d}_{r}\right)_{i} . &
\end{array}
$$

If one defines, following Davini [2],

$$
S_{r a}=\frac{\nabla \wedge \boldsymbol{d}_{r} \cdot \boldsymbol{d}_{a}}{n},
$$

then from (2.16),

$$
S_{r a}=\boldsymbol{L}_{a} \cdot \boldsymbol{d}_{r}
$$

The matrix $\left(S_{r a}\right)$ is called the dislocation density tensor: the columns of $S$ represent the components of the Lie brackets with respect to the lattice vector fields $\ell_{1}(\cdot), \ell_{2}(\cdot), \ell_{3}(\cdot)$, and the rows of $S$ represent (weighted) components of the Burgers' vectors, with respect to the same fields.

\section{Elastic deformation, integrability condition, right-invariant fields}

Suppose that two sets of linearly independent lattice vector fields, $\ell_{1}(\cdot), \ell_{2}(\cdot), \ell_{3}(\cdot)$ and $\tilde{\ell}_{1}(\cdot)$, $\tilde{\ell}_{2}(\cdot), \tilde{\ell}_{3}(\cdot)$ are given on $\mathbb{R}^{3}$. If there exists a mapping $\boldsymbol{\psi}: \mathbb{R}^{3} \rightarrow \mathbb{R}^{3}$ such that

$$
\tilde{\boldsymbol{\ell}}_{a}(\boldsymbol{\psi}(\boldsymbol{x}))=\nabla \boldsymbol{\psi}(\boldsymbol{x}) \boldsymbol{\ell}_{a}(\boldsymbol{x}), \quad a=1,2,3, \boldsymbol{x} \in \mathbb{R}^{3},
$$

then we say that the two sets of fields are elastic related via the deformation $\boldsymbol{\psi}$. One may calculate from (3.1) that

$$
\begin{aligned}
& \tilde{\boldsymbol{d}}_{a}(\boldsymbol{\psi}(\boldsymbol{x}))=[\nabla \boldsymbol{\psi}(\boldsymbol{x})]^{-T} \boldsymbol{d}_{a}(\boldsymbol{x}), \quad a=1,2,3, \quad \boldsymbol{x} \in \mathbb{R}^{3} \\
& \tilde{\boldsymbol{L}}_{b}(\boldsymbol{\psi}(\boldsymbol{x}))=\nabla \boldsymbol{\psi}(\boldsymbol{x}) \boldsymbol{L}_{b}(\boldsymbol{x}), \quad b=1,2,3, \quad \boldsymbol{x} \in \mathbb{R}^{3},
\end{aligned}
$$


where $\tilde{\boldsymbol{L}}_{3}(\cdot), \tilde{\boldsymbol{L}}_{1}(\cdot), \tilde{\boldsymbol{L}}_{(\cdot)}$ are the Lie brackets corresponding to the pairs of fields $\tilde{\boldsymbol{\ell}}_{1}(\cdot), \tilde{\ell}_{2}(\cdot)$; $\tilde{\ell}_{2}(\cdot), \tilde{\ell}_{3}(\cdot) ; \tilde{\ell}_{3}(\cdot) \tilde{\ell}_{1}(\cdot)$, etc.. Then from $(2.18)$

$$
\tilde{S}_{r a}(\boldsymbol{\psi}(\boldsymbol{x}))=S_{r a}(\boldsymbol{x}), \quad r, a,=1,2,3, \quad \boldsymbol{x} \in \mathbb{R}^{3},
$$

where $\left(\tilde{S}_{r a}(\cdot)\right)$ is the dislocation density tensor deriving from the fields $\tilde{\ell}_{1}(\cdot), \tilde{\ell}_{2}(\cdot), \tilde{\ell}_{3}(\cdot)$. So the dislocation density tensor is unchanged by elastic deformation, thus it is a measure of inelastic deformation. For an exhaustive list of differential functions which have this property (of 'elastic invariance') see Davini and Parry [3], Parry and Šilhavý [4], Olver [1].

Recall that we are interested in crystals where one can assign a strain energy per unit volume of the crystal given relevant kinematic variables. For a perfect crystal (without defects), one traditionally assumes that a strain energy function of the form

$$
w\left(\ell_{1}, \ell_{2}, \ell_{3}\right) \equiv w\left(\left\{\boldsymbol{\ell}_{a}\right\}\right)
$$

where vectors $\ell_{1}, \ell_{2}, \ell_{3} \in \mathbb{R}^{3}$ are prescribed, relates to the potential energy per unit volume of an infinite lattice

$$
L \equiv\left\{\boldsymbol{x}: \boldsymbol{x}=n_{a} \ell_{a}, n_{a} \in \mathbb{Z}, a=1,2,3\right\}
$$

One imagines that the vectors $\boldsymbol{\ell}_{1}, \boldsymbol{\ell}_{2}, \boldsymbol{\ell}_{3}$ are the local values (at a point $\boldsymbol{x}_{0}$, say) of fields $\boldsymbol{\ell}_{1}(\cdot), \boldsymbol{\ell}_{2}(\cdot), \boldsymbol{\ell}_{3}(\cdot)$, and that the lattice $L$ represents the local structure at $\boldsymbol{x}_{0}$. In the perfect crystal case, one assumes that the fields $\boldsymbol{\ell}_{1}(\cdot), \boldsymbol{\ell}_{2}(\cdot), \boldsymbol{\ell}_{3}(\cdot)$ are constant in space, so $\boldsymbol{\ell}_{a}\left(\boldsymbol{x}_{0}\right) \equiv \boldsymbol{\ell}_{a}$, for all $\boldsymbol{x}_{0} \in \mathbb{R}^{3}$. The perfect lattice $L$ may be obtained from the lattice vector fields as follows: starting at the origin, iterate along the lattice vector fields to reach the points $e^{\ell_{1}}=$ $\ell_{1}, e^{\ell_{2}}=\ell_{2}, e^{\ell_{3}}=\ell_{3}$ (calculating from $(2.1)$ ). Also iterate along $-\ell_{1}(\cdot),-\ell_{2}(\cdot),-\ell_{3}(\cdot)$ to reach $e^{-\ell_{1}}=-\ell_{1}, e^{-\ell_{2}}=-\ell_{2}, e^{-\ell_{3}}=-\ell_{3}$. Iterate again, starting at the points $\pm \boldsymbol{\ell}_{1}, \pm \boldsymbol{\ell}_{2}, \pm \boldsymbol{\ell}_{3}$, and continue in this way to obtain $L$ as the set which consists of all points obtained by successive iteration along $\pm \boldsymbol{\ell}_{1}(\cdot), \pm \boldsymbol{\ell}_{2}(\cdot), \pm \boldsymbol{\ell}_{3}(\cdot)$, starting at the origin. The geometrical symmetries of $L$ transfer to 'material' symmetries of the strain energy function if one assumes that $w$ depends only on $L$, not on the lattice vectors which are employed to describe $L-$ thus if $\left\{\boldsymbol{\ell}_{a}\right\}$ and $\left\{\ell_{a}^{\prime}\right\}$ are local values of lattice vector fields which produce the same lattice $L$ according to the procedure outlined above, then this geometrical symmetry transfers to the requirement that $w\left(\left\{\boldsymbol{\ell}_{a}\right\}\right)=w\left(\left\{\boldsymbol{\ell}_{a}^{\prime}\right\}\right)$.

We shall be concerned with the generalization of the above procedure to cases where the crystal has defects and the constitutive form of the energy density reflects the existence of 
defects. In fact we consider what seems to be the simplest extension of (3.4) that allows for defects and suppose that

$$
w=w\left(\left\{\ell_{a}\right\}, S\right),
$$

where $S$ is the dislocation density tensor. We take it that the values $\left\{\boldsymbol{\ell}_{a}\right\}, S$ which occur as arguments of $w$ are the values of the vector fields $\left\{\boldsymbol{\ell}_{a}(\cdot)\right\}$, and corresponding dislocation density tensor $S(\cdot)$, at some point $\boldsymbol{x}_{0} \in \mathbb{R}^{3}$. Note that if $S \neq 0$ then from (2.17), $\nabla \wedge \boldsymbol{d}_{a} \neq \mathbf{0}$ for some index $a$, so that the lattice vector fields $\boldsymbol{\ell}_{a}(\cdot)$, whose local values are prescribed to be $\boldsymbol{\ell}_{a}$, cannot be constant in space.

Since the constitutive form of $w$ provides no information regarding higher derivatives of $S(\cdot)$, we made the simple assumption that the fields $\left\{\boldsymbol{\ell}_{a}(\cdot)\right\}$ are such that $S(\cdot)$ is constant in space. This is a critical assumption that allows us to introduce a certain Lie group structure - to see how this structure arises first of all suppose that fields $\left\{\boldsymbol{\ell}_{a}(\cdot)\right\}$ are given and ask whether or not the differential system

$$
\boldsymbol{\ell}_{a}(\boldsymbol{\psi}(\boldsymbol{x}, \boldsymbol{y}))=\nabla_{1} \boldsymbol{\psi}(\boldsymbol{x}, \boldsymbol{y}) \boldsymbol{\ell}_{a}(\boldsymbol{x}), \quad a=1,2,3, \boldsymbol{x}, \boldsymbol{y} \in \mathbb{R}^{3}
$$

has a solution for $\boldsymbol{\psi}: \mathbb{R}^{3} \times \mathbb{R}^{3} \rightarrow \mathbb{R}^{3}$. (In $\left.(3.7)\right), \nabla_{1} \boldsymbol{\psi}(\boldsymbol{x}, \boldsymbol{y})$ denotes the gradient of $\boldsymbol{\psi}$ with respect to its first argument).

Note that if one fixes $\boldsymbol{y}$ in (3.7) and puts $\boldsymbol{\psi}(\boldsymbol{x}) \equiv \boldsymbol{\psi}(\boldsymbol{x}, \boldsymbol{y})$, then from (3.1), (3.7) states that there is an elastic deformation $\boldsymbol{\psi}$ which maps the set of fields $\left\{\ell_{a}(\cdot)\right\}$ to itself - in this respect the fields $\left\{\boldsymbol{\ell}_{a}(\cdot)\right\}$ have a certain 'self-similarity' and from (3.3), $S(\boldsymbol{\psi}(\boldsymbol{x}))=S(\boldsymbol{x})$, which implies that $S$ is constant (depending on the range of the function $\psi$ ).

In fact, according to Pontryagin [5], (3.7) has a solution for $\boldsymbol{\psi}$ provided the corresponding dislocation density tensor $S$ is constant in space (we shall say that $S$ is 'uniform', henceforward). Moreover, any solution of (3.7) is associative, so

$$
\boldsymbol{\psi}(\boldsymbol{x}, \boldsymbol{\psi}(\boldsymbol{y}, \boldsymbol{z}))=\boldsymbol{\psi}(\boldsymbol{\psi}(\boldsymbol{x}, \boldsymbol{y}), \boldsymbol{z}), \quad \boldsymbol{x}, \boldsymbol{y}, \boldsymbol{z} \in \mathbb{R}^{3},
$$

and (3.7) has a unique solution which satisfies the additional conditions

$$
\boldsymbol{\psi}(\boldsymbol{x}, \mathbf{0})=\boldsymbol{x}=\boldsymbol{\psi}(\mathbf{0}, \boldsymbol{x}), \quad \boldsymbol{x} \in \mathbb{R}^{3} .
$$

(We shall not be concerned, in this paper, with lattice vector fields such that solutions $\psi$ of (3.7) are not defined on all of $\mathbb{R}^{3} \times \mathbb{R}^{3}$ ). In addition, for each $\boldsymbol{x} \in \mathbb{R}^{3}$, this exists on $\boldsymbol{x}^{-1} \in \mathbb{R}^{3}$ such that

$$
\boldsymbol{\psi}\left(\boldsymbol{x}, \boldsymbol{x}^{-1}\right)=\boldsymbol{\psi}\left(\boldsymbol{x}^{-1}, \boldsymbol{x}\right)=\mathbf{0} .
$$


Thus, our assumption that $S$ is uniform leads to the existence of a function $\psi$ which expresses a self similarity of the vector fields $\left\{\boldsymbol{\ell}_{a}(\cdot)\right\}$ via (3.7), and which has the properties (3.8), (3.9), (3.10).

Properties (3.8), (3.9), (3.10) are those necessary in order that $\mathbb{R}^{3}$, with binary operation $\boldsymbol{\psi}$, be regarded as a Lie group, denoted $G$. (The function $\psi$ defines a 'multiplication'of points in $\left.\mathbb{R}^{3}\right)$. It is the condition that $S$ is uniform that is the 'integrability condition' which guarantees that (3.7) has a solution for $\boldsymbol{\psi}$.

Finally, in this section, suppose just that $\boldsymbol{\psi}$ satisfies (3.7). Then by differentiating with respect to $\boldsymbol{x}$,

$$
\nabla_{1} \boldsymbol{\psi}(\boldsymbol{x}, \boldsymbol{\psi}(\boldsymbol{y}, \boldsymbol{z}))=\nabla_{1} \boldsymbol{\psi}(\boldsymbol{\psi}(\boldsymbol{x}, \boldsymbol{y}), \boldsymbol{z}) \nabla_{1} \boldsymbol{\psi}(\boldsymbol{x}, \boldsymbol{y}),
$$

so putting $\boldsymbol{x}=\mathbf{0}$,

$$
\nabla_{1} \boldsymbol{\psi}(\mathbf{0}, \boldsymbol{\psi}(\boldsymbol{y}, \boldsymbol{z})) \boldsymbol{e}_{a}=\nabla_{1} \boldsymbol{\psi}(\boldsymbol{y}, \boldsymbol{z})\left[\nabla_{1} \boldsymbol{\psi}(0, \boldsymbol{y}) \boldsymbol{e}_{a}\right]
$$

Thus, if one defines

$$
\boldsymbol{\ell}_{a}(\boldsymbol{p})=\nabla_{1} \boldsymbol{\psi}(\mathbf{0}, \boldsymbol{p}) \boldsymbol{e}_{a}
$$

one sees that (3.7) holds for this choice of $\boldsymbol{\ell}_{a}(\cdot)$. That is to say, given $\boldsymbol{\psi}$, one can construct fields $\boldsymbol{\ell}_{a}(\cdot)$ which satisfy $(3.7)$. Let $\boldsymbol{\ell}(\cdot)$ be an arbitrary field which satisfies $(3.7)$, so

$$
\ell(\boldsymbol{\psi}(\boldsymbol{x}, \boldsymbol{y}))=\nabla_{1} \boldsymbol{\psi}(\boldsymbol{x}, \boldsymbol{y}) \boldsymbol{\ell}(\boldsymbol{x}) .
$$

Then putting $\boldsymbol{x}=\mathbf{0}$, and $\boldsymbol{\ell}(\mathbf{0})=\nu_{i} \boldsymbol{e}_{i}, \nu_{i} \in \mathbb{R}, \quad i=1,2,3$,

$$
\begin{aligned}
\ell(\boldsymbol{y}) & =\nabla_{1} \boldsymbol{\psi}(\mathbf{0}, \boldsymbol{y}) \nu_{i} \boldsymbol{e}_{i} \\
& =\nu_{i}\left[\nabla \boldsymbol{\psi}(0, \boldsymbol{y}) \boldsymbol{e}_{i}\right]
\end{aligned}
$$

which shows that the particular fields (3.14) provide the basis for the set of all vector fields $\ell(\cdot)$ which satisfy (3.7). The set of all vector fields which satisfy (3.7), given $\boldsymbol{\psi}$, is called the set of all right-invariant fields corresponding to the multiplication function $\psi$.

\section{Flow along right-invariant fields, discrete subgroups of Lie groups, solvable groups}

Let $\ell(\cdot)$ be a right-invariant field, satisfying (3.7). Consider the flow $\boldsymbol{x}$ generated by $\boldsymbol{\ell}(\cdot)$, so that $\boldsymbol{x}\left(t, \boldsymbol{x}_{0}\right)$ satisfies

$$
\frac{d}{d t} \boldsymbol{x}\left(t, \boldsymbol{x}_{0}\right)=\boldsymbol{\ell}\left(\boldsymbol{x}\left(t, \boldsymbol{x}_{0}\right)\right), \quad \boldsymbol{x}\left(0, \boldsymbol{x}_{0}\right)=\boldsymbol{x}_{0}
$$


Suppose that $\boldsymbol{\ell}(0)=\boldsymbol{\ell} \in \mathbb{R}^{3}$, so that from (3.7)

$$
\boldsymbol{\ell}(\boldsymbol{y})=\nabla_{a} \boldsymbol{\psi}(0, \boldsymbol{y}) \boldsymbol{\ell}(0) \equiv \nabla_{1} \boldsymbol{\psi}(0, \boldsymbol{y}) \boldsymbol{\ell}
$$

Then if $\boldsymbol{\ell}$ is given, the field $\boldsymbol{\ell}(\cdot)$ is known, given $\boldsymbol{\psi}$. So if $\boldsymbol{\ell} \in \mathbb{R}^{3}$ and $\boldsymbol{\psi}$ are given, $\boldsymbol{x}\left(t, \boldsymbol{x}_{0}\right)$ is determined via (4.1), so the mapping $\exp (t \ell)$, and the point $e^{t \ell}$ are well defined.

\section{Lemma 1.}

$$
\exp (t \ell) \boldsymbol{x}=\boldsymbol{\psi}\left(e^{t \ell}, \boldsymbol{x}\right)
$$

Proof Note that $e^{t \ell}$ solves

$$
\frac{d}{d t} \boldsymbol{x}(t, \mathbf{0})=\boldsymbol{\ell}(\boldsymbol{x}(t, \mathbf{0})), \quad \boldsymbol{x}(0, \mathbf{0})=\mathbf{0} .
$$

So

$$
\frac{d}{d t} \boldsymbol{\psi}\left(e^{t \ell}, \boldsymbol{x}\right)=\nabla_{1} \boldsymbol{\psi}\left(e^{t \ell}, \boldsymbol{x}\right) \boldsymbol{\ell}\left(e^{t \ell}\right)=\boldsymbol{\ell}\left(\boldsymbol{\psi}\left(e^{t \ell}, \boldsymbol{x}\right)\right)
$$

by (3.7). Therefore $\boldsymbol{\psi}\left(e^{t \boldsymbol{\ell}}, \boldsymbol{x}\right)$ is the unique solution of (4.1) which takes the value $\boldsymbol{x}$ when $t=0$. The result follows by definition of $\exp (t \boldsymbol{\ell}) \boldsymbol{x}$.

This standard lemma asserts that 'flow along a right-invariant field is equivalent to group multiplication' - it is important for our work.

Now we return to the question of what discrete set of points should be associated with the arguments $\left\{\boldsymbol{\ell}_{a}\right\}, S$ of an energy density for defective crystals. We assume that $S$ is the value of a uniform dislocation density tensor deriving from fields $\boldsymbol{\ell}_{a}(\cdot)$ with $\boldsymbol{\ell}_{a}(\mathbf{0})=\boldsymbol{\ell}_{a}$. Given that $S$ is uniform, there exists a Lie group composition function $\boldsymbol{\psi}: \mathbb{R}^{3} \times \mathbb{R}^{3} \rightarrow \mathbb{R}^{3}$ such that (3.7) holds, and (3.8), (3.9), (3.10) are satisfied. Thus the fields $\boldsymbol{\ell}_{a}(\cdot)$ are right-invariant with respect to $\boldsymbol{\psi}$, and flow along these right-invariant fields corresponds to group multiplication by the elements $e^{\ell_{1}}, e^{\ell_{2}}, e^{\ell_{3}}$, or their inverses. Iterating the flow corresponds to successive multiplication by $e^{\ell_{1}}, e^{\ell_{2}}, e^{\ell_{3}}$ and their inverses, and the collection of all these products is a subgroup $D$ of the Lie group $G$ that is $\mathbb{R}^{3}$ with multiplication function $\boldsymbol{\psi}$. We write

$$
D=g p\left(e^{\ell_{1}}, e^{\ell_{2}}, e^{\ell_{3}}\right)
$$

for the group generated by the three elements (points of $\mathbb{R}^{3}$ ) $e^{\ell_{1}}, e^{\ell_{2}}, e^{\ell_{3}}$ and their inverses. The set of points $D$ is the generalization, to defective crystals, of the lattices $L$, for perfect crystals - however note that $L$ has an additional property which is not so far, guaranteed by the construction of the set of points $D$, as outlined above. That property is the 'crystallographic 
restriction' which is used as a starting point in classical work to list and classify point sets deemed to be appropriate as models of perfect real crystals. We adopt that restriction for defective crystals too, that is we assume that there is a minimum separation between the points of $D$, i.e. we assume that $D$ is a discrete subgroup of the relevant Lie group. Note that Auslander, Green and Hahn [6] have listed those three dimensional Lie groups which have discrete subgroups, and given canonical forms of generators of those discrete subgroups. To understand their results, we need to introduce some formal Lie group machinery.

First, as a convenient notation, we denote multiplication of elements in $G$ by juxtaposition, and put

$$
\psi(\boldsymbol{x}, \boldsymbol{y})=\boldsymbol{x y}, \quad \boldsymbol{x}, \boldsymbol{y} \in G .
$$

Define the commutator of group elements $\boldsymbol{x}, \boldsymbol{y}$, by

$$
(\boldsymbol{x}, \boldsymbol{y})=\boldsymbol{x}^{-1} \boldsymbol{y}^{-1} \boldsymbol{x} \boldsymbol{y}
$$

For the Lie groups that are relevant to this paper, the underlying manifold is $\mathbb{R}^{3}$ and we associate coordinates $x_{1}, x_{2}, x_{3} \in \mathbb{R}$ with a group element $\boldsymbol{x}=x_{i} \boldsymbol{e}_{i}$ by introducing a basis $\left\{\boldsymbol{e}_{1}, \boldsymbol{e}_{2}, \boldsymbol{e}_{3}\right\}$ of $\mathbb{R}^{3}$. Here, the Lie algebra of $G$ is denoted $\mathfrak{g}$ - it is $\mathbb{R}^{3}$ with binary operation

$$
[\boldsymbol{x}, \boldsymbol{y}]=C_{i j k} x_{j} y_{k} \boldsymbol{e}_{i}, \quad \boldsymbol{x}, \boldsymbol{y} \in \mathbb{R}^{3},
$$

when the structure constants $C_{i j k}, i, j, k,=1,2,3$ are defined by

$$
C_{i j k}=\frac{\partial^{2} \psi_{i}}{\partial x_{j} \partial y_{k}}(\mathbf{0}, \mathbf{0})-\frac{\partial^{2} \psi_{i}}{\partial x_{k} \partial y_{j}}(\mathbf{0}, \mathbf{0})
$$

where $\boldsymbol{\psi}(\boldsymbol{x}, \boldsymbol{y})=\psi_{i}(\boldsymbol{x}, \boldsymbol{y}) \boldsymbol{e}_{i}$. The structure constants are connected to the dislocation density tensor via the relation

$$
C_{i j k} \ell_{r j}(\mathbf{0}) \ell_{s k}(\mathbf{0})=\varepsilon_{p r s} S_{k p} \ell_{k i}(\mathbf{0})
$$

where $\boldsymbol{\ell}_{r}(\mathbf{0})=\ell_{r j}(\mathbf{0}) \boldsymbol{e}_{j}$, see Elzanowski and Parry [7].

Let $G^{\prime}$ be the derived group of $G$ - this is the group generated by all commutators of elements of $G$, also called the commutator subgroup. One writes

$$
G^{\prime} \equiv(G, G)
$$

and understands that the notation does not imply that each element of $G^{\prime}$ is a commutator of elements $\boldsymbol{x}, \boldsymbol{y} \in G$, only that each element of $G^{\prime}$ is a product of such terms. Analogously one 
may introduce the group $\left(G^{\prime}, G\right)$ as that generated by commutators of elements $\boldsymbol{x} \in G^{\prime}, \boldsymbol{y} \in G$, etc.. It turns out, according to Auslander, Green and Hahn [6], that there are two class of three dimensional Lie groups which have non trivial discrete subgroups: this is the nilpotent group, which has the property that

$$
\left(G^{\prime}, G\right)=\mathbf{0}
$$

and there are the solvable groups, which have the property that

$$
\left(G^{\prime}, G^{\prime}\right)=\mathbf{0}
$$

We have dealt with the nilpotent case in a previous paper, so we shall be concerned here with the case that (4.14) holds, so that commutators of group elements commute with each other.

At the level of Lie algebra, let

$$
\mathfrak{g}^{\prime} \equiv[\mathfrak{g}, \mathfrak{g}]
$$

denote the set of all elements $\sum_{i}\left[\boldsymbol{g}_{i}, \boldsymbol{h}_{i}\right], \boldsymbol{g}_{i}, \boldsymbol{h}_{i} \in \mathfrak{g}$. This is called the derived algebra of $\mathfrak{g}$. It is a fact that $G$ is nilpotent if and only if

$$
\left[\mathfrak{g}^{\prime}, \mathfrak{g}\right]=\mathbf{0}
$$

and that $G$ is solvable if and only if

$$
\left[\mathfrak{g}^{\prime}, \mathfrak{g}^{\prime}\right]=\mathbf{0}
$$

in the cases with which we shall be concerned, in this paper.

According to Auslander, Green and Hahn [6], Jacobson [8], a basis $\boldsymbol{X}, \boldsymbol{Y}, \boldsymbol{Z}$ of $\mathbb{R}^{3}$ may be chosen so that, in the nilpotent case,

$$
[\boldsymbol{X}, \boldsymbol{Y}]=\boldsymbol{Z},[\boldsymbol{X}, \boldsymbol{Z}]=\mathbf{0},[\boldsymbol{Y}, \boldsymbol{Z}]=\mathbf{0},
$$

and in the solvable case

$$
[\boldsymbol{X}, \boldsymbol{Y}]=0,[\boldsymbol{X}, \boldsymbol{Z}]=\alpha \boldsymbol{X}+\beta \boldsymbol{Y},[\boldsymbol{Y}, \boldsymbol{Z}]=\gamma \boldsymbol{X}+\delta \boldsymbol{Y},
$$

where $\alpha, \beta, \gamma, \delta \in \mathbb{R}$ satisfy

$$
\alpha \delta=\beta \gamma \neq 0
$$


These relations give the structure constants of the algebra with respect to the basis vectors $\boldsymbol{X}, \boldsymbol{Y}, \boldsymbol{Z}$, since from (4.9) the structure constants with respect to any basis $\boldsymbol{e}_{1}, \boldsymbol{e}_{2}, \boldsymbol{e}_{3}$, are given by

$$
\left[\boldsymbol{e}_{i}, \boldsymbol{e}_{j}\right]=C_{k i j} \boldsymbol{e}_{k} .
$$

So by (4.11), knowing the structure constants, we can find the dislocation density tensor, for the cases where the relevant Lie group ( or algebras) is nilpotent or solvable, and we reiterate that these are the only cases where the iteration procedure described above leads to a discrete subgroup (of $G$ ).

For the nilpotent case, the structure of the discrete subgroup $D$ has been studied in Cermelli and Parry [9] and the symmetries of that structure have been found in Parry and Sigrist [10]. The solvable case falls naturally into two cases, and Auslander, Green and Hahn denote the corresponding groups $S_{1}$ and $S_{2}$. Discrete subgroups of $S_{1}$, and their symmetries, have been investigated in Nicks and Parry [11]. Here we study the discrete subgroups of $S_{2}$ and their symmetries. Much material in Nicks and Parry [11] is also relevant to the case at hand - we quote results from that paper without proof, but explain technical terms in order to facilitate comprehension of the methods involved. There are some qualitative differences between the two cases, nevertheless.

\section{The solvable groups $S_{1}$ and $S_{2}$}

Auslander, Green and Hahn [6] treat these groups, which for our purposes consist of $\mathbb{R}^{3}$ with a corresponding multiplication rule, by associating with each point $\boldsymbol{x} \in \mathbb{R}^{3}$ a $4 \times 4$ matrix which we denote by $r_{m}(\boldsymbol{x})$ (i.e. we represent $\boldsymbol{x}$ as a $4 \times 4$ matrix). The multiplication rule for $S_{1}, S_{2}$ follows from the usual law for matrix multiplication once it is recognized that the mapping $r_{m}(\cdot)$ is one to one from $\mathbb{R}^{3}$ to $r_{m}\left(\mathbb{R}^{3}\right)$. Thus

$$
r_{m}(\boldsymbol{\psi}(\boldsymbol{x}, \boldsymbol{y})) \equiv r_{m}(\boldsymbol{x y})=r_{m}(\boldsymbol{x}) r_{m}(\boldsymbol{y}),
$$

defines $\boldsymbol{\psi}$, since it is a fact that $r_{m}(\boldsymbol{x}) r_{m}(\boldsymbol{y}) \in r_{m}\left(\mathbb{R}^{3}\right)$, and $r_{m}^{-1}(\cdot)$ exists. We call $r_{m}\left(\mathbb{R}^{3}\right)$, with matrix multiplication as group composition function, the group $S_{m}$.

$$
\begin{aligned}
& \text { Explicitly, } r_{m}(\cdot) \text { is defined by }
\end{aligned}
$$

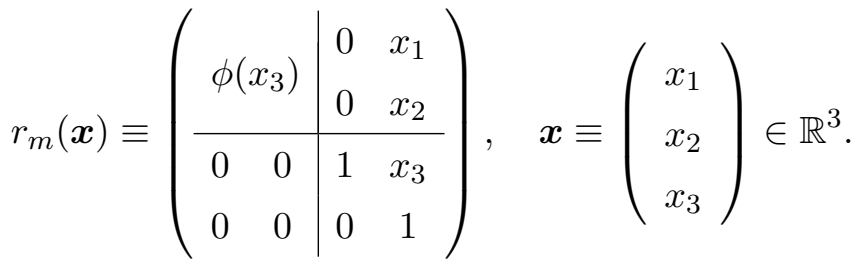


where $\phi\left(x_{3}\right) \in S L_{2}(\mathbb{R}), \phi(1) \in S L_{2}(\mathbb{Z})$ and $\left\{\phi\left(x_{3}\right): x_{3} \in \mathbb{R}\right\}$ is a one parameter subgroup of the unimodular group. This means that $\phi\left(x_{3}\right)$ is a $2 \times 2$ matrix with real entries and determinant 1 , that $\phi(1)$ is a $2 \times 2$ matrix with integer entries, determinant 1 , and that

$$
\phi(x) \phi(y)=\phi(x+y), \quad \boldsymbol{x}, \boldsymbol{y} \in \mathbb{R} .
$$

Hence $\phi(0)=\mathbb{I}_{2}$, the $2 \times 2$ identity matrix. The one parameter subgroups, $\left\{\phi\left(x_{3}\right): x_{3} \in \mathbb{R}\right\}$ of $S L_{2}(\mathbb{R})$ which have $\phi(1) \in S L_{2}(\mathbb{Z})$ fall into two classes and they correspond to the matrix groups (5.2), $S_{1}$ and $S_{2}$. This classification depends on the eigenvalues of $\phi(1)$. Let us write

$$
\phi(x)=\left(\begin{array}{cc}
a(x) & b(x) \\
c(x) & d(x)
\end{array}\right), \quad \text { and } \quad \theta \equiv \phi(1)=\left(\begin{array}{cc}
a(1) & b(1) \\
c(1) & d(1)
\end{array}\right) \equiv\left(\begin{array}{cc}
a & b \\
c & d
\end{array}\right) .
$$

The eigenvalues, $\lambda$, of $\theta$ satisfy the characteristic equation $\lambda^{2}-\operatorname{tr}(\theta) \lambda+1=0$ where $\operatorname{tr}(\theta)=a+d$ denotes the trace of the matrix $\theta$. Therefore $\lambda=\frac{1}{2}\left(a+d \pm \sqrt{(a+d)^{2}-4}\right)$ so that the eigenvalues of $\theta$ are $\lambda, 1 / \lambda$ and the possibilities for $\theta \in S L_{2}(\mathbb{Z})$ can be classified as follows:

1. If $|\operatorname{tr}(\theta)|>2$ then the eigenvalues of $\theta$ are real and distinct (and $\theta$ is called a hyperbolic element of $S L_{2}(\mathbb{R})$ ). In the case that $\operatorname{tr}(\theta)=a+d>2$ the corresponding Lie group is denoted $S_{1}$, and this case has been treated in Nicks and Parry [11]. When $a+d<2$, it can be shown that $\theta$ cannot lie on a one parameter subgroup of the unimodular group, so this case is not of interest, here;

2. If $|\operatorname{tr}(\theta)|=2$ then the eigenvalues of $\theta$ are real and equal (and $\theta$ is called a parabolic element of $\left.S L_{2}(\mathbb{R})\right)$. In the case that $\operatorname{tr}(\theta)=a+d=2$, the corresponding Lie group is nilpotent and this case has been treated in Cermelli and Parry [9], Parry and Sigrist [10];

3. If $|\operatorname{tr}(\theta)|<2$ then the eigenvalues of $\theta$ are complex conjugates (and $\theta$ is called an elliptic element of $\left.S L_{2}(\mathbb{R})\right)$.

In this paper we shall consider the cases that remain to be treated, where $\theta$ is such that $\operatorname{tr}(\theta) \in\{-2,-1,0,1\}$ and call this class of groups $S_{2}$. We shall consider the discrete subgroups of $S_{2}$ and their symmetries.

Now, differentiating (5.3) with respect to $y$ and putting $y=0$ and similarly for $x$ we see that the one parameter subgroup $\phi(x)$ of $S L_{2}(\mathbb{R})$ satisfies

$$
\phi^{\prime}(x)=\phi(x) \phi^{\prime}(0)=\phi^{\prime}(0) \phi(x),
$$

where' denotes $\frac{d}{d x}$. Let us define

$$
\phi^{\prime}(0)=\mathcal{A}=\left(\begin{array}{ll}
a^{\prime}(0) & b^{\prime}(0) \\
c^{\prime}(0) & d^{\prime}(0)
\end{array}\right) .
$$


Then from (5.5) we have

$$
\phi(x)=\mathrm{e}^{\mathcal{A} x}=\sum_{j=0}^{\infty} \mathcal{A}^{j} \frac{x^{j}}{j !} .
$$

Since $\phi(x) \in S L_{2}(\mathbb{Z}), a(x) d(x)-b(x) c(x)=1$ and differentiating this relation with respect to $x$ and putting $x=0$ we see that $a^{\prime}(0)+d^{\prime}(0)=0$ since $\phi(0)=\mathbb{I}_{2}$. Hence

$$
\mathcal{A}^{2}=-\operatorname{det}(\mathcal{A}) \mathbb{I}_{2} .
$$

For any matrix $\mathcal{A}$ satisfying (5.8)

$$
\mathrm{e}^{\mathcal{A}}= \begin{cases}(\cosh k) \mathbb{I}_{2}+\left(\frac{\sinh k}{k}\right) \mathcal{A} & \text { if } \operatorname{det}(\mathcal{A})<0, k \equiv \sqrt{-\operatorname{det}(\mathcal{A})} \\ (\cos k) \mathbb{I}_{2}+\left(\frac{\sin k}{k}\right) \mathcal{A} & \text { if } \operatorname{det}(\mathcal{A})>0, k \equiv \sqrt{\operatorname{det}(\mathcal{A})} \\ \mathbb{I}_{2}+\mathcal{A} & \text { if } \operatorname{det}(\mathcal{A})=0 ;\end{cases}
$$

and thus since $\operatorname{tr}(\mathcal{A})=0$,

$$
a+d=\operatorname{tr}\left(\mathrm{e}^{\mathcal{A}}\right)=\left\{\begin{array}{ll}
2 \cosh k & \text { if } \operatorname{det}(\mathcal{A})<0, \\
2 \cos k & \text { if } \operatorname{det}(\mathcal{A})>0, \\
2 & \text { if } \operatorname{det}(\mathcal{A})=0
\end{array} \quad \text { where } k=\sqrt{|\operatorname{det}(\mathcal{A})|}\right.
$$

Recall that we are interested in the cases where $a+d \in\{-2,-1,0,1\}$, so that $\operatorname{det} \mathcal{A}>0$ for the class of groups $S_{2}$. In these cases $\theta-\mathbb{I}_{2}$ is invertible and $\phi: \mathbb{R} \rightarrow S L_{2}(\mathbb{R})$ is given by $\phi(x)=\mathrm{e}^{\mathcal{A} x}=(\cos (k x)) \mathbb{I}_{2}+\left(\frac{\sin (k x)}{k}\right) \mathcal{A}$, where $k= \begin{cases}\pi n, \quad n= \pm 1 \bmod 2 \text { when } a+d=-2 \\ \frac{2 \pi n}{3}, n= \pm 1 \bmod 3 \text { when } a+d=-1 \\ \frac{\pi n}{2}, \quad n= \pm 1 \bmod 4 \text { when } a+d=0 \\ \frac{\pi n}{3}, \quad n= \pm 1 \bmod 6 \text { when } a+d=1 .\end{cases}$

Then, when $a+d \in\{-1,0,1\}$,

$\theta=\phi(1)=\left(\begin{array}{ll}a & b \\ c & d\end{array}\right)=\frac{1}{2}(a+d) \mathbb{I}_{2}+\left(\frac{\sin k}{k}\right) \mathcal{A} \Rightarrow \mathcal{A}=\left(\frac{k}{\sin k}\right)\left(\begin{array}{cc}\frac{1}{2}(a-d) & b \\ c & -\frac{1}{2}(a-d)\end{array}\right)$,

and when $a+d=-2, \phi(1)=-\mathbb{I}_{2}$ and $\mathcal{A}$ is any traceless $2 \times 2$ matrix with determinant $k^{2}=n^{2} \pi^{2}, n$ is an odd integer. 
Let $\boldsymbol{\psi}: \mathbb{R}^{3} \times \mathbb{R}^{3} \rightarrow \mathbb{R}^{3}$ denote the group operation in $S_{2}$, then from (5.1) one can compute that

$$
\boldsymbol{\psi}(\boldsymbol{x}, \boldsymbol{y})=\boldsymbol{x}+\left(a\left(x_{3}\right) y_{1}+b\left(x_{3}\right) y_{2}\right) \boldsymbol{e}_{1}+\left(c\left(x_{3}\right) y_{1}+d\left(x_{3}\right) y_{2}\right) \boldsymbol{e}_{2}+y_{3} \boldsymbol{e}_{3},
$$

where $\boldsymbol{x}=x_{i} \boldsymbol{e}_{i} \equiv\left(x_{1}, x_{2}, x_{3}\right)^{T}, \boldsymbol{y}=y_{i} \boldsymbol{e}_{i} \equiv\left(y_{1}, y_{2}, y_{3}\right)^{T}, T$ denotes transpose, $\left\{\boldsymbol{e}_{1}, \boldsymbol{e}_{2}, \boldsymbol{e}_{3}\right\}$ is a basis of $\mathbb{R}^{3}$ and the summation convention operates.

From (4.9) and (4.10) one computes that the Lie bracket on $\mathfrak{s}_{2}$, the Lie algebra of $S_{2}$, is given by

$$
[\boldsymbol{x}, \boldsymbol{y}]=\left(a^{\prime}(0) \boldsymbol{x} \wedge \boldsymbol{y} \cdot \boldsymbol{e}_{2}-b^{\prime}(0) \boldsymbol{x} \wedge \boldsymbol{y} \cdot \boldsymbol{e}_{1}\right) \boldsymbol{e}_{1}+\left(c^{\prime}(0) \boldsymbol{x} \wedge \boldsymbol{y} \cdot \boldsymbol{e}_{2}-d^{\prime}(0) \boldsymbol{x} \wedge \boldsymbol{y} \cdot \boldsymbol{e}_{1}\right) \boldsymbol{e}_{2}
$$

Thus

$$
\left[\boldsymbol{e}_{1}, \boldsymbol{e}_{2}\right]=\mathbf{0},\left[\boldsymbol{e}_{1}, \boldsymbol{e}_{3}\right]=-a^{\prime}(0) \boldsymbol{e}_{1}-c^{\prime}(0) \boldsymbol{e}_{2},\left[\boldsymbol{e}_{2}, \boldsymbol{e}_{3}\right]=-b^{\prime}(0) \boldsymbol{e}_{1}-d^{\prime}(0) \boldsymbol{e}_{2}
$$

Comparing with (4.19) we see that $\alpha=-a^{\prime}(0), \beta=-c^{\prime}(0), \gamma=-b^{\prime}(0), \delta=-d^{\prime}(0)$, so that the remark that $\alpha \delta-\beta \gamma \neq 0$ in order that the relevant Lie group be solvable is equivalent to the requirement that $\operatorname{det} \mathcal{A} \neq 0$.

One can also compute the dislocation density tensor $\mathcal{S}$. First

$$
\nabla_{1} \boldsymbol{\psi}(\mathbf{0}, \boldsymbol{x})=\left(\begin{array}{ccc}
1 & 0 & \mathcal{A}\left(\begin{array}{c}
x_{1} \\
x_{2}
\end{array}\right) \\
0 & 1 & 1 \\
0 & 0 & 1
\end{array}\right)
$$

and hence the right invariant vector fields $\boldsymbol{\ell}_{a}(\boldsymbol{x})=\nabla_{1} \boldsymbol{\psi}(\mathbf{0}, \boldsymbol{x}) \boldsymbol{e}_{a}$ are given by

$$
\boldsymbol{\ell}_{1}(\boldsymbol{x})=\boldsymbol{e}_{1}, \quad \boldsymbol{\ell}_{2}(\boldsymbol{x})=\boldsymbol{e}_{2}, \quad \boldsymbol{\ell}_{3}(\boldsymbol{x})=\left(a^{\prime}(0) x_{1}+b^{\prime}(0) x_{2}\right) \boldsymbol{e}_{1}+\left(c^{\prime}(0) x_{1}+d^{\prime}(0) x_{2}\right) \boldsymbol{e}_{2}+\boldsymbol{e}_{3} .
$$

Then the dual lattice vector fields are

$$
\boldsymbol{d}_{1}(\boldsymbol{x})=\boldsymbol{e}_{1}-\left(a^{\prime}(0) x_{1}+b^{\prime}(0) x_{2}\right) \boldsymbol{e}_{3}, \quad \boldsymbol{d}_{2}(\boldsymbol{x})=\boldsymbol{e}_{2}-\left(c^{\prime}(0) x_{1}+d^{\prime}(0) x_{2}\right) \boldsymbol{e}_{3}, \quad \boldsymbol{d}_{3}(\boldsymbol{x})=\boldsymbol{e}_{3},
$$

and from (2.17) and (5.18) the components of the dislocation density tensor are

$$
\left(\frac{\nabla \wedge \boldsymbol{d}_{a} \cdot \boldsymbol{d}_{b}}{\boldsymbol{d}_{1} \cdot \boldsymbol{d}_{2} \wedge \boldsymbol{d}_{3}}\right)=\left(\begin{array}{ccc}
-b^{\prime}(0) & -d^{\prime}(0) & 0 \\
a^{\prime}(0) & c^{\prime}(0) & 0 \\
0 & 0 & 0
\end{array}\right)=\frac{k}{\sin k}\left(\begin{array}{ccc}
-b & \frac{1}{2}(a-d) & 0 \\
\frac{1}{2}(a-d) & c & 0 \\
0 & 0 & 0
\end{array}\right)
$$

in the cases where $a+d \in\{-1,0,1\}$. 
Note that the correspondence between the dislocation density tensor, which is uniquely determined by the matrix $\mathcal{A}$, and the quantity $\theta \equiv \phi(1)=e^{\mathcal{A}}$ is infinitely many to one, in general. For $\theta$ determines $\operatorname{tr}(\theta) \equiv a+d=2 \cos k$, so that , in (5.19), $k$ may take a countable infinity of values, $k= \pm k_{0}+2 m \pi, 0 \leq k_{0} \leq \pi, m \in \mathbb{Z}$, say. So for $a+d \in\{-1,0,1\}$,

$$
\mathcal{A}=\frac{k_{0}}{\sin k_{0}}\left(\begin{array}{cc}
\frac{1}{2}(a-d) & b \\
c & -\frac{1}{2}(a-d)
\end{array}\right)+m \frac{2 \pi}{\sin k_{0}}\left(\begin{array}{cc}
\frac{1}{2}(a-d) & b \\
c & -\frac{1}{2}(a-d)
\end{array}\right), \quad m \in \mathbb{Z},
$$

where $a+d=2 \cos k_{0}, a d-b c=1$.

Also, if $a+d=-2$, then $\theta=-\mathbb{I}_{2}$ and

$$
\mathcal{A}=\left(\begin{array}{cc}
\lambda & \mu \\
\nu & -\lambda
\end{array}\right), \quad-\lambda^{2}-\mu \nu=(2 n+1)^{2} \pi^{2}, \quad n \in \mathbb{Z} .
$$

See Rossmann [12] for further comments on the properties of the matrix exponential function.

\section{The discrete subgroups of $S_{2}$}

\subsection{The discrete subgroups $\mathcal{D}$}

According to Auslander, Green and Hahn [6], in the cases where $\theta-\mathbb{I}_{2} \in S L_{2}(\mathbb{Z})$ is invertible; that is when $\operatorname{tr}(\theta) \in\{-2,-1,0,1\}$, the discrete subgroup $\mathcal{D} \subset S_{2}$ is isomorphic (via $r_{m}$ above) to a discrete subgroup $\mathcal{D}_{m} \subset S_{m}$ and $\mathcal{D}_{m}$ is generated by three elements

$A \equiv r_{m}\left(\boldsymbol{e}_{3}\right)=\left(\begin{array}{cc|cc}\theta & 0 & 0 \\ 0 & 0 & 1 & 1 \\ 0 & 0 & 0 & 1\end{array}\right), B \equiv r_{m}\left(\boldsymbol{e}_{1}\right)=\left(\begin{array}{cccc}1 & 0 & 0 & 1 \\ 0 & 1 & 0 & 0 \\ 0 & 0 & 1 & 0 \\ 0 & 0 & 0 & 1\end{array}\right), C \equiv r_{m}\left(\boldsymbol{e}_{2}\right)=\left(\begin{array}{cccc}1 & 0 & 0 & 0 \\ 0 & 1 & 0 & 1 \\ 0 & 0 & 1 & 0 \\ 0 & 0 & 0 & 1\end{array}\right)$.

One may identify canonical forms of the matrix $\theta$ in (6.1), corresponding to the four choices of $\operatorname{tr} \theta \in\{-2,-1,0,1\}$, by finding representatives of the conjugacy classes of $\theta$ in $S L_{2}(\mathbb{R})$. (Mapping $\theta$ to $P^{-1} \theta P, P \in S L_{2}(\mathbb{R})$, amounts to a change of coordinates $x_{1}, x_{2}$ which preserves the orientation of the basis vectors). This is done as follows. First the set of eigenvalues of $\theta$, $\left\{e^{i p}, e^{-i p}\right\}$ say, is determined by $\operatorname{tr}(\theta)$ since $\operatorname{det}(\theta)=1$, and one may specify that $p>0$. Then one can choose $P \in S L_{2}(\mathbb{R})$ such that

$$
P^{-1} \theta P=\left\{\begin{array}{rr}
\left(\begin{array}{rr}
\cos p & \sin p \\
-\cos p & \cos p
\end{array}\right), & \text { if } \quad b>0, \\
\left(\begin{array}{rr}
\cos p & -\sin p \\
\sin p & \cos p
\end{array}\right), \quad \text { if } \quad b<0 .
\end{array}\right.
$$


(Take $P$ to have columns equal to scaled real and imaginary parts of the eigenvectors of $\theta$, in some order, and note that the sign of the 12 element of $\theta$, denoted $b$, is the same as the sign of the 12 element of any conjugate of $\theta$ in $S L_{2}(\mathbb{R})$. See Conrad [13] for details). In the three cases $\operatorname{tr}(\theta) \in\{-1,0,1\}, b \neq 0$ because there is no corresponding matrix in $S L_{2}(\mathbb{Z})$ where $b=0$, and so there are two conjugacy classes in each of these three cases - one can write down representatives of the two classes simply by identifying two matrices in $S L_{2}(\mathbb{Z})$ with the correct trace, one with $b>0$ and the other with $b<0$. In the case that $\operatorname{tr}(\theta)=-2$, there is only one conjugacy class which consists of the single element $\theta=-\mathbb{I}_{2}$ (when $\theta$ is required to lie on a one parameter subgroup of $S L_{2}(\mathbb{R})$ ).

In each case,

$$
\theta^{2}=\operatorname{tr}(\theta) \theta-\mathbb{I}_{2}
$$

and

- If $\operatorname{tr}(\theta)=-1, \theta$ is conjugate to either $\left(\begin{array}{ll}-1 & 1 \\ -1 & 0\end{array}\right)(b>0)$ or $\left(\begin{array}{ll}0 & -1 \\ 1 & -1\end{array}\right)(b<0)$. Also $\theta^{3}=\mathbb{I}_{2}$,

- If $\operatorname{tr}(\theta)=0, \theta$ is conjugate to either $\left(\begin{array}{rr}0 & 1 \\ -1 & 0\end{array}\right)(b>0)$ or $\left(\begin{array}{rr}0 & -1 \\ 1 & 0\end{array}\right)(b<0)$. Also $\theta^{4}=\mathbb{I}_{2}$,

- If $\operatorname{tr}(\theta)=1, \theta$ is conjugate to either $\left(\begin{array}{rr}1 & 1 \\ -1 & 0\end{array}\right)(b>0)$ or $\left(\begin{array}{rr}0 & -1 \\ 1 & 1\end{array}\right)(b<0)$. Also $\theta^{6}=\mathbb{I}_{2}$,

- If $\operatorname{tr}(\theta)=-2, \theta$ is conjugate to $-\mathbb{I}_{2}$ (in fact $\theta=-\mathbb{I}_{2}$ ). Also $\theta^{2}=\mathbb{I}_{2}$.

Let $(\boldsymbol{x}, \boldsymbol{y})=\boldsymbol{x}^{-1} \boldsymbol{y}^{-1} \boldsymbol{x} \boldsymbol{y}$ denote the commutator of elements $\boldsymbol{x}, \boldsymbol{y} \in \mathcal{D}$ where we write $\boldsymbol{x} \boldsymbol{y}=$ $\boldsymbol{\psi}(\boldsymbol{x}, \boldsymbol{y})$. Similarly, let $(X, Y)=X^{-1} Y^{-1} X Y$ denote the commutator of elements $X, Y \in \mathcal{D}_{m}$. One can then compute that

$$
(A, B)=B^{1-d} C^{c}, \quad(A, C)=B^{b} C^{1-a}, \quad(B, C)=0 .
$$

Any element of $\mathcal{D}_{m}$ can be expressed as a product of the form

$$
d_{m}=A^{\alpha_{1}} B^{\beta_{1}} C^{\gamma_{1}} A^{\alpha_{2}} B^{\beta_{2}} C^{\gamma_{2}} \cdots A^{\alpha_{r}} B^{\beta_{r}} C^{\gamma_{r}}
$$


with $\alpha_{i}, \beta_{i}, \gamma_{i} \in\{0 \pm 1\}, i=1, \ldots, r$. Noting that $B C=C B$ and that one can write

$$
B^{\beta} C^{\gamma} A^{\alpha}=A^{\alpha} B^{\beta} C^{\gamma}\left(B^{\beta} C^{\gamma}, A^{\alpha}\right)=A^{\alpha} B^{m} C^{n} \quad \text { where }\left(\begin{array}{c}
m \\
n
\end{array}\right)=\theta^{-\alpha}\left(\begin{array}{l}
\beta \\
\gamma
\end{array}\right) \text {, }
$$

we can rewrite (6.4) as

$$
d_{m}=A^{\alpha_{1}+\alpha_{2}+\cdots+\alpha_{r}} B^{M} C^{N}
$$

for some $M, N \in \mathbb{Z}$. Since $\mathcal{D}_{m}$ contains $A^{Q} B^{M} C^{N}$ for any $Q, M, N \in \mathbb{Z}$ a general element $d_{m}=A^{Q} B^{M} C^{N} \in \mathcal{D}_{m}$ has the representation

$$
\left(\begin{array}{c|ccc}
\theta^{Q} & 0 & \theta^{Q}\left(\begin{array}{c}
M \\
N
\end{array}\right) \\
\hline 0 & 0 & 1 & Q \\
0 & 0 & 0 & 1
\end{array}\right)
$$

Let $\boldsymbol{x} \in \mathbb{R}^{3}$ be such that $r_{m}(\boldsymbol{x})=A^{Q} B^{M} C^{N}, Q, M, N \in \mathbb{Z}$. Then

$$
\boldsymbol{x}=\left(\begin{array}{c}
\theta^{Q}\left(\begin{array}{c}
M \\
N
\end{array}\right) \\
Q
\end{array}\right)
$$

Since $\theta \in S L_{2}(\mathbb{Z})$ it is clear that $r_{m}^{-1}\left(\mathcal{D}_{m}\right)=\mathcal{D}=\left(\mathbb{Z}^{3}, \psi\right)$ so that the discrete subgroup of $S_{2}$ with which we are concerned in the cases where $\theta$ has trace $-2,-1,0$ or 1 is the cubic lattice $\mathbb{Z}^{3}$ with group multiplication given by (5.13).

\subsection{Composition and commutators in $\mathcal{D}$}

Suppose that $d_{1}=A^{x_{3}} B^{x_{1}} C^{x_{2}}$ and $d_{2}=A^{y_{3}} B^{y_{1}} C^{y_{2}}$ are elements of $\mathcal{D}_{m}$ (i.e. $x_{i}, y_{i} \in \mathbb{Z}, i=$ $1,2,3)$ with corresponding elements $\boldsymbol{d}_{1}=r_{m}^{-1}\left(d_{1}\right)$ and $\boldsymbol{d}_{2}=r_{m}^{-1}\left(d_{2}\right)$ respectively. Then one can compute from the form of an element of $\mathcal{D}_{m}$ given by (6.7) that

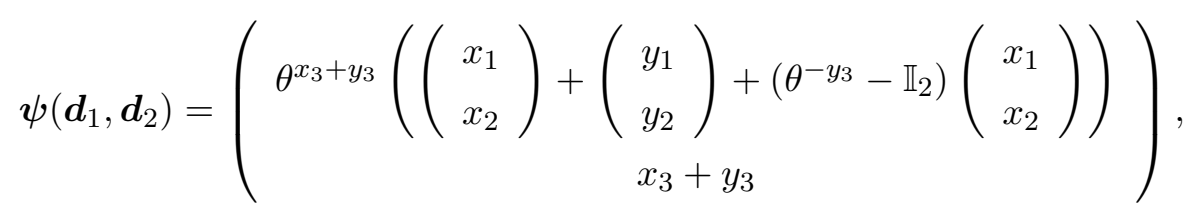

where

$$
\begin{aligned}
\left(\theta^{-y_{3}}-\mathbb{I}_{2}\right) & =\left(\theta^{-1}-\mathbb{I}_{2}\right)\left(\mathbb{I}_{2}+\theta^{-1}+\theta^{-2}+\cdots+\theta^{-y_{3}+1}\right) \\
& =\left(\theta^{-1}-\mathbb{I}_{2}\right) \sum_{j=0}^{p-1} \theta^{-j}, \text { where } 0 \leq p<n, p=y_{3} \quad \bmod n \text { for } n=2,3,4,6,
\end{aligned}
$$


where $n$ is the order of $\theta$.

We can then see that

$$
\boldsymbol{d}_{1}^{-1}=-\left(\begin{array}{l}
x_{1} \\
x_{2} \\
x_{3}
\end{array}\right)
$$

and hence

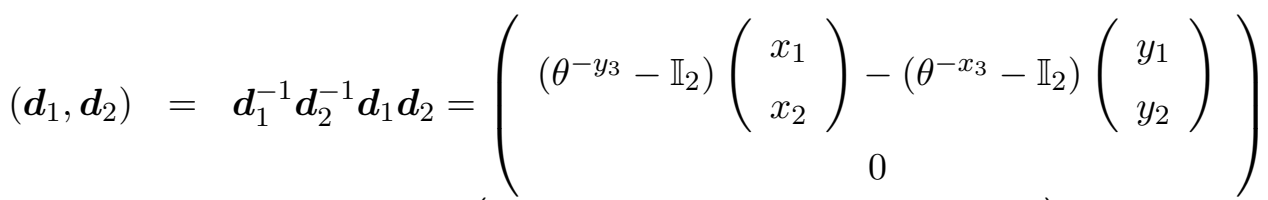

$$
\begin{aligned}
& =\left(\begin{array}{c}
\left(\theta^{-1}-\mathbb{I}_{2}\right)\left(\sum_{j=0}^{p-1} \theta^{-j}\left(\begin{array}{c}
x_{1} \\
x_{2}
\end{array}\right)-\sum_{j=0}^{q-1} \theta^{-j}\left(\begin{array}{l}
y_{1} \\
y_{2}
\end{array}\right)\right) \\
0
\end{array}\right) \\
& \left.=\left(\begin{array}{cc}
d-1 & -b \\
-c & a-1
\end{array}\right)\left(\begin{array}{l}
w_{1} \\
w_{2}
\end{array}\right)\right)
\end{aligned}
$$

where $q=x_{3} \bmod n, p=y_{3} \bmod n$ and $n=2,3,4,6$ is the order of $\theta$. Then

$$
\left(d_{1}, d_{2}\right)=\left(B^{d-1} C^{-c}\right)^{w_{1}}\left(B^{-b} C^{a-1}\right)^{w_{2}}=(B, A)^{w_{1}}(C, A)^{w_{2}} .
$$

So the commutator (or derived) subgroup $\mathcal{D}_{m}^{\prime}$ of $\mathcal{D}_{m}$ is the subgroup generated by $(B, A)$ and $(C, A)$.

Let $r_{m}(\boldsymbol{u})=(B, A)$ and $r_{m}(\boldsymbol{v})=(C, A)$, and let $\mathcal{D}_{m}^{\prime}=r_{m}\left(\mathcal{D}^{\prime}\right)$. Then $\mathcal{D}^{\prime}$ is generated by $\boldsymbol{u}$ and $\boldsymbol{v}$. From (5.13), if $x_{3}=y_{3}=0$ then $\boldsymbol{\psi}(\boldsymbol{x}, \boldsymbol{y})=\boldsymbol{x}+\boldsymbol{y}$ and since $\boldsymbol{u}$ and $\boldsymbol{v}$ are commutators they have third component equal to zero. Thus $r_{m}(\alpha \boldsymbol{u}+\beta \boldsymbol{v})=(B, A)^{\alpha}(C, A)^{\beta}, \alpha, \beta \in \mathbb{Z}$ and the points of $\mathcal{D}^{\prime}$ coincide with the sublattice of $\mathbb{Z}^{3}$ generated (additively) by $\boldsymbol{u}$ and $\boldsymbol{v}$. Let $\langle\boldsymbol{u}, \boldsymbol{v}\rangle$ denote the integer span of the vectors $\boldsymbol{u}, \boldsymbol{v} \in \mathbb{Z}^{3}$. For convenience we write

$$
\boldsymbol{u}=\left(\begin{array}{c}
d-1 \\
-c
\end{array}\right), \boldsymbol{v}=\left(\begin{array}{c}
-b \\
a-1
\end{array}\right) \quad \text { instead of } \quad \boldsymbol{u}=\left(\begin{array}{c}
d-1 \\
-c \\
0
\end{array}\right), \quad \boldsymbol{v}=\left(\begin{array}{c}
-b \\
a-1 \\
0
\end{array}\right)
$$

and let $\{\boldsymbol{u}, \boldsymbol{v}\}$ denote the $2 \times 2$ matrix with columns $\boldsymbol{u}, \boldsymbol{v}$, so that $\{\boldsymbol{u}, \boldsymbol{v}\}=\left(\theta^{-1}-\mathbb{I}_{2}\right)\left\{\boldsymbol{e}_{1}, \boldsymbol{e}_{2}\right\}$.

Notice that $\operatorname{det}\left(\theta^{-1}-\mathbb{I}_{2}\right)=2-(a+d)$ so that in the case that $a+d=1$, the points of $\mathcal{D}^{\prime}$ are just $\mathbb{Z}^{2}=\left\{(r, s, t) \in \mathbb{Z}^{3}: t=0\right\}$. In the case where $a+d=-2$ so that $\theta=-\mathbb{I}^{2}, \boldsymbol{u}=(-2,0)^{T}$ 
and $\boldsymbol{v}=(0,-2)^{T}$ and hence the points of $\mathcal{D}^{\prime}$ are $(2 \mathbb{Z})^{2}=\left\{(r, s, t) \in \mathbb{Z}^{3}: t=0, r, s \in 2 \mathbb{Z}\right\}$. In the cases where $a+d=0$ or -1 , the points of $\mathcal{D}^{\prime}$ coincide with a proper sublattice of $\mathbb{Z}^{2}$ with unit cell of area $(2-a-d)$.

\section{$7 \quad$ Generators of $\mathcal{D}$}

Let $\boldsymbol{g}_{1}, \boldsymbol{g}_{2}, \boldsymbol{g}_{3}$ be elements of $\mathcal{D}$. We wish to determine conditions on the choice of those three elements which are necessary and sufficient that the group generated by $\boldsymbol{g}_{1}, \boldsymbol{g}_{2}, \boldsymbol{g}_{3}$, with composition function (5.13) denoted

$$
\mathcal{G}=g p\left(\boldsymbol{g}_{1}, \boldsymbol{g}_{2}, \boldsymbol{g}_{3}\right),
$$

equals $\mathcal{D}$. The analogous problem, in the case of the class of solvable groups $S_{1}$, was treated in Nicks and Parry [11]. The calculations in that paper transfer without difficulty to the case at hand, so here we summarize relevant facts and conclusions, without giving proofs, so that the main ideas may be clearly seen.

Let

$$
\boldsymbol{g}_{i m} \equiv r_{m}\left(\boldsymbol{g}_{i}\right)=A^{\alpha_{i}} B^{\beta_{i}} C^{\gamma_{i}}, \quad \alpha_{i}, \beta_{i}, \gamma_{i} \in \mathbb{Z}, \quad i=1,2,3,
$$

where there is no summation over $i$. If $\mathcal{G}=\mathcal{D}$, then $\mathcal{G}_{m} \equiv g p\left(\boldsymbol{g}_{1 m}, \boldsymbol{g}_{2 m}, \boldsymbol{g}_{3 m}\right)$, with matrix multiplication as group operation, equals $\mathcal{D}_{m}$. But if $\boldsymbol{g}_{m} \in \mathcal{G}_{m}$, then

$$
\boldsymbol{g}_{m}=\boldsymbol{g}_{1 m}^{\varepsilon_{1}} \boldsymbol{g}_{2 m}^{\nu_{1}} \boldsymbol{g}_{3 m}^{\mu_{1}} \boldsymbol{g}_{1 m}^{\varepsilon_{2}} \boldsymbol{g}_{2 m}^{\nu_{2}} \boldsymbol{g}_{3 m}^{\mu_{2}} \ldots \boldsymbol{g}_{1 m}^{\varepsilon_{r}} \boldsymbol{g}_{2 m}^{\nu_{r}} \boldsymbol{g}_{3 m}^{\mu_{r}}
$$

where $\varepsilon_{i}, \nu_{i}, \mu_{i} \in\{0, \pm 1\}, i=1 \ldots r$. This expression may be rewritten, via (6.3), (6.5), (7.2), as

$$
\boldsymbol{g}_{m}=A^{\alpha_{1}\left(\varepsilon_{1}+\cdots+\varepsilon_{r}\right)+\alpha_{2}\left(\nu_{1}+\cdots+\nu_{r}\right)+\alpha_{3}\left(\mu_{1} \cdots+\mu_{r}\right)} B^{M} C^{N},
$$

for some $M, N \in \mathbb{Z}$ whose values depend on $\alpha_{i}, \beta_{i}, \gamma_{i}, \varepsilon_{i}, \nu_{i}, \mu_{i}$ for $i=1,2,3$. Since $A \in \mathcal{G}_{m}$ and $A^{\ell} \neq B^{m} C^{n}$ for any $\ell, m, n, \in \mathbb{Z} \backslash\{0\}$, it follows that there are integers $\varepsilon \equiv \varepsilon_{1}+\cdots+\varepsilon_{r}, \nu \equiv$ $\nu_{1}+\cdots+\nu_{r}, \mu \equiv \mu_{1}+\cdots+\mu_{r}$, such that $\alpha_{1} \varepsilon+\alpha_{2} \nu+\alpha_{3} \mu=1$. Therefore $\alpha_{1}, \alpha_{2}, \alpha_{3}$ are relatively prime integers. Let $h c f(a, b \ldots c)$ denote the positive highest common factor of the set of integers $\{a, b \ldots c\}$. Then

$$
h c f\left(\alpha_{1}, \alpha_{2}, \alpha_{3}\right)=1 \text {, }
$$

and we state the following Lemma without proof (see [11] for details). 
Lemma 2. Let $\boldsymbol{g}_{1 m}, \boldsymbol{g}_{2 m}, \boldsymbol{g}_{3 m}$ be given by (7.2), let $\mathcal{G}_{m}=g p\left(\boldsymbol{g}_{1 m}, \boldsymbol{g}_{2 m}, \boldsymbol{g}_{3 m}\right)$ and suppose that $h c f\left(\alpha_{1}, \alpha_{2}, \alpha_{3}\right)=1$. Then there is a set of generators of $\mathcal{G}_{m}$, denoted $\boldsymbol{g}_{1 m}^{\prime}, \boldsymbol{g}_{2 m}^{\prime}, \boldsymbol{g}_{3 m}^{\prime}$, such that

$$
\boldsymbol{g}_{1 m}^{\prime}=A B^{\beta_{1}^{\prime}} C^{\gamma_{1}^{\prime}}, \boldsymbol{g}_{2 m}^{\prime}=B^{\beta_{2}^{\prime}} C^{\gamma_{2}^{\prime}}, \boldsymbol{g}_{3 m}^{\prime}=B^{\beta_{3}^{\prime}} C^{\gamma_{3}^{\prime}}, \quad \beta_{i}^{\prime}, \gamma_{i}^{\prime} \in \mathbb{Z}, \quad i=1,2,3 .
$$

To facilitate some of the following calculations, we introduce the notation

$$
x^{y}=y^{-1} x y, \quad x, y \in \mathcal{G}
$$

for the conjugate of an element $\boldsymbol{x} \in \mathcal{G}$. Then we have:

$$
\left(x^{y}\right)^{z}=x^{(y z)},(x y)^{z}=x^{z} y^{z},\left(x^{y}\right)^{-1}=\left(x^{-1}\right)^{y}, x^{x^{-1} y}=x^{y},(x, y)^{z}=\left(x^{z}, y^{z}\right),
$$

for $\boldsymbol{x}, \boldsymbol{y}, \boldsymbol{z} \in \mathcal{G}$.

Also define

$$
\boldsymbol{x}^{n \boldsymbol{y}} \equiv\left(\boldsymbol{x}^{n}\right)^{\boldsymbol{y}}=\left(\boldsymbol{x}^{\boldsymbol{y}}\right)^{n}, n \in \mathbb{Z}, \boldsymbol{x}, \boldsymbol{y}=\mathcal{G} .
$$

Now commutators commute in $\mathcal{G}$, and since $(\boldsymbol{x}, \boldsymbol{y})^{\boldsymbol{z}}=\left(\boldsymbol{x}^{\boldsymbol{z}}, \boldsymbol{y}^{\boldsymbol{z}}\right)$, terms of the form $(\boldsymbol{x}, \boldsymbol{y})^{\boldsymbol{z}}$ also commute in $\mathcal{G}, \boldsymbol{x}, \boldsymbol{y}, \boldsymbol{z} \in \mathcal{G}$. Furthermore one can show that

$$
(\boldsymbol{x}, \boldsymbol{y})^{\boldsymbol{w} z}=(\boldsymbol{x}, \boldsymbol{y})^{\boldsymbol{z} \boldsymbol{w}}, \quad \boldsymbol{x}, \boldsymbol{y}, \boldsymbol{z}, \boldsymbol{w} \in \mathcal{G}
$$

This allows us to define $(\boldsymbol{x}, \boldsymbol{y})^{P}$, where $P$ is a polynomial with integer coefficients in the generators of $\mathcal{G}=g p\left(\boldsymbol{g}_{1}, \boldsymbol{g}_{2}, \boldsymbol{g}_{3}\right)$, and their inverses, as follows. Let $P$ be written (uniquely) in the form $P=\sum_{\boldsymbol{\alpha}} \varepsilon_{\boldsymbol{\alpha}} p_{\boldsymbol{\alpha}}$, where $\varepsilon_{\boldsymbol{\alpha}}$ is nonzero only for a finite number of choices of multi-index $\boldsymbol{\alpha}=\left(\alpha_{1}, \alpha_{2}, \alpha_{3}\right), \varepsilon_{\boldsymbol{\alpha}} \in \mathbb{Z}, \alpha_{1}, \alpha_{2}, \alpha_{3} \in \mathbb{Z}$, where $p_{\boldsymbol{\alpha}} \equiv \boldsymbol{g}_{1}^{\alpha_{1}} \boldsymbol{g}_{2}^{\alpha_{2}} \boldsymbol{g}_{3}^{\alpha_{3}}$, and let $P^{\prime}=\sum_{\boldsymbol{\alpha}} \varepsilon_{\boldsymbol{\alpha}}^{\prime} p_{\boldsymbol{\alpha}}^{\prime}$ be similarly defined. Then put

$$
(\boldsymbol{x}, \boldsymbol{y})^{P}=\prod_{\boldsymbol{\alpha}}(\boldsymbol{x}, \boldsymbol{y})^{\varepsilon \boldsymbol{\alpha} p \boldsymbol{\alpha}},
$$

and also put

$$
\left\{(\boldsymbol{x}, \boldsymbol{y})^{P}\right\}^{P^{\prime}}=\prod_{\boldsymbol{\alpha}^{\prime}}\left\{(\boldsymbol{x}, \boldsymbol{y})^{P}\right\}^{\varepsilon_{\boldsymbol{\alpha}}^{\prime} p_{\boldsymbol{\alpha}}^{\prime}} .
$$

It is straightforward to show that, with these definitions,

$$
\begin{aligned}
& (\boldsymbol{x}, \boldsymbol{y})^{n P}=\left\{(\boldsymbol{x}, \boldsymbol{y})^{n}\right\}^{P}=\left\{(\boldsymbol{x}, \boldsymbol{y})^{P}\right\}^{n}, \\
& (\boldsymbol{x}, \boldsymbol{y})^{P}(\boldsymbol{x}, \boldsymbol{y})^{P^{\prime}}=(\boldsymbol{x}, \boldsymbol{y})^{P+P^{\prime}}, \\
& \left\{(\boldsymbol{x}, \boldsymbol{y})^{P}\right\}^{P^{\prime}}=\left\{(\boldsymbol{x}, \boldsymbol{y})^{P^{\prime}}\right\}^{P}=(\boldsymbol{x}, \boldsymbol{y})^{P P^{\prime}} .
\end{aligned}
$$

Note that $(\boldsymbol{x}, \boldsymbol{y})^{P}$ is a product of commutators, via (7.8), (7.9), (7.11), so it is an element of the commutator subgroup $\mathcal{G}^{\prime} \equiv(\mathcal{G}, \mathcal{G})$. 
Lemma 3. (i) Let $\boldsymbol{g}$ be arbitrary element of $\mathcal{G}=g p\left(\boldsymbol{g}_{1}, \boldsymbol{g}_{2}, \boldsymbol{g}_{3}\right)$. Then $\boldsymbol{g}$ can be written in the form

$$
\boldsymbol{g}_{1}^{\alpha} \boldsymbol{g}_{2}^{\beta} \boldsymbol{g}_{3}^{\gamma}\left(\boldsymbol{g}_{1}, \boldsymbol{g}_{2}\right)^{P_{3}}\left(\boldsymbol{g}_{2}, \boldsymbol{g}_{3}\right)^{P_{1}}\left(\boldsymbol{g}_{3}, \boldsymbol{g}_{1}\right)^{P_{2}}
$$

where $P_{1}, P_{2}, P_{3}$ are polynomials in $\boldsymbol{g}_{1}, \boldsymbol{g}_{2}, \boldsymbol{g}_{3}$ and their inverses.

(ii) Let $\boldsymbol{g}^{\prime} \in \mathcal{G}^{\prime} \equiv(\mathcal{G}, \mathcal{G})$. Then $\boldsymbol{g}^{\prime}$ can be written in the form

$$
\left(\boldsymbol{g}_{1}, \boldsymbol{g}_{2}\right)^{P_{3}}\left(\boldsymbol{g}_{2}, \boldsymbol{g}_{3}\right)^{P_{1}}\left(\boldsymbol{g}_{3}, \boldsymbol{g}_{1}\right)^{P_{2}}
$$

which is a product of terms of the form

$$
\left(\boldsymbol{g}_{i}, \boldsymbol{g}_{j}\right)^{\boldsymbol{g}_{1}^{\alpha} \boldsymbol{g}_{2}^{\beta} \boldsymbol{g}_{3}^{\gamma}}, \quad \alpha, \beta, \gamma \in \mathbb{Z}, \quad i, j=1,2,3
$$

Statement (i) in that lemma is a generalization of the expression $\boldsymbol{x}=m_{1} \boldsymbol{\ell}_{1}+m_{2} \boldsymbol{\ell}_{2}+m_{3} \boldsymbol{\ell}_{3}$, $m_{1}, m_{2}, m_{3} \in \mathbb{Z}$ for the general element $\boldsymbol{x}$ of a perfect lattice with basis $\boldsymbol{\ell}_{1}, \boldsymbol{\ell}_{2}, \boldsymbol{\ell}_{3}$ and a generalization of Mal'cev's expression $\boldsymbol{g}=\boldsymbol{g}_{1}^{m_{1}} \boldsymbol{g}_{2}^{m_{2}} \boldsymbol{g}_{3}^{m_{3}}, m_{1}, m_{2}, m_{3} \in \mathbb{Z}$ for the elements of a discrete subgroup of a nilpotent Lie group in terms of a canonical basis $\boldsymbol{g}_{1}, \boldsymbol{g}_{2}, \boldsymbol{g}_{3}$, to the case at hand where the relevant Lie group is solvable. The statement that (ii) holds may be found in Bachmuth [14].

\section{Conditions that $\mathcal{G}=\mathcal{D}$}

We wish to determine the conditions that $\mathcal{G} \equiv g p\left(\boldsymbol{g}_{1}, \boldsymbol{g}_{2}, \boldsymbol{g}_{3}\right)$ equals $\mathcal{D}$, and a necessary condition for this is that $\mathcal{G}^{\prime}=\mathcal{D}^{\prime}$. First, we consider $\mathcal{G}^{\prime}$, using Lemma 2 (ii), which shows that $\mathcal{G}^{\prime}$ is generated by $\left(\boldsymbol{g}_{i}, \boldsymbol{g}_{j}\right)^{\boldsymbol{h}}$, for all $\boldsymbol{h}$ of the form $\boldsymbol{g}_{1}^{\alpha} \boldsymbol{g}_{2}^{\beta} \boldsymbol{g}_{3}^{\gamma}, \alpha, \beta, \gamma \in \mathbb{Z}, i<j, i, j=1,2,3$. By calculating $\left(\boldsymbol{g}_{i}, \boldsymbol{g}_{j}\right)^{\boldsymbol{h}}$ explicitly, it is shown in [11] that

$$
\mathcal{G}^{\prime}=\left\langle\left(\boldsymbol{g}_{i}, \boldsymbol{g}_{j}\right), \theta\left(\boldsymbol{g}_{i}, \boldsymbol{g}_{j}\right) ; \quad i<j, i, j=1,2,3\right\rangle
$$

Furthermore, if the generators of $\mathcal{G}$ are such that (7.6) holds, then

$$
\left.\mathcal{G}^{\prime}=\langle\boldsymbol{a}, \boldsymbol{b}), \theta \boldsymbol{a}, \theta \boldsymbol{b}\right\rangle,
$$

when $\boldsymbol{a} \equiv\left(\boldsymbol{g}_{1}, \boldsymbol{g}_{2}\right), \boldsymbol{b} \equiv\left(\boldsymbol{g}_{1}, \boldsymbol{g}_{3}\right)$.

Now $\mathcal{D}^{\prime}$ is known, from section 6.2, to be generated (additively) by $\boldsymbol{u}, \boldsymbol{v} \in \mathbb{Z}^{3}$ given by (6.14). It is therefore an exercise to linear algebra to determine the conditions on $\boldsymbol{a} \equiv\left(\boldsymbol{g}_{1}, \boldsymbol{g}_{2}\right), \boldsymbol{b} \equiv$ 
$\left(\boldsymbol{g}_{1}, \boldsymbol{g}_{3}\right)$ that guarantee $\mathcal{G}^{\prime}=\mathcal{D}^{\prime}$. It turns out that these conditions depend on the parameters $\beta_{2}^{\prime}, \gamma_{2}^{\prime}, \beta_{2}^{\prime}, \gamma_{2}^{\prime}$ that appear in (7.6), see [11]. Putting

$$
\boldsymbol{\tau}_{1} \equiv\left(\begin{array}{c}
\beta_{2}^{\prime} \\
\gamma_{2}^{\prime}
\end{array}\right), \quad \tau_{2} \equiv\left(\begin{array}{c}
\beta_{3}^{\prime} \\
\gamma_{3}^{\prime}
\end{array}\right), \quad \tau_{3} \equiv \theta \boldsymbol{\tau}_{1}, \quad \tau_{4} \equiv \theta \boldsymbol{\tau}_{2},
$$

and letting the components of $\boldsymbol{\tau}_{i}, i=1,2,3,4$, be $\left(\begin{array}{c}\tau_{1 i} \\ \tau_{2 i}\end{array}\right)$, the conditions are divisibility conditions on the quantities $\tau_{\alpha i}, \alpha=1,2, i=1,2,3,4$ :

$$
\begin{aligned}
& h c f\left(\tau_{11}, \tau_{12}, \tau_{13}, \tau_{14}\right)=h c f\left(\tau_{21}, \tau_{22}, \tau_{23}, \tau_{24}\right)=1, \\
& h c f\left(\boldsymbol{\tau}_{i} \wedge \boldsymbol{\tau}_{j} ; i<j, i, j=1,2,3,4,\right)=1,
\end{aligned}
$$

where $\boldsymbol{\tau}_{i} \wedge \boldsymbol{\tau}_{j} \equiv \tau_{1 i} \tau_{2 j}-\tau_{1 j} \tau_{2 i}, i, j=1,2,3,4$. These conditions, (8.4) and (8.5), are also sufficient that $\mathcal{G}^{\prime}=\mathcal{D}^{\prime}$. In fact, it is shown in [11], that (8.4) and (8.5) are also necessary and sufficient that $\mathcal{G}=\mathcal{D}$.

\section{Conclusion}

The calculations above complete the determination of the symmetries of discrete subgroups of three dimensional Lie groups, meant to model crystals with a continuum strain energy density per unit volume that has the constitutive form $w=w\left(\left\{\boldsymbol{\ell}_{a}\right), S\right)$. Finding these 'symmetries' corresponds to finding the set of all (sets of three) generators of the relevant Lie groups, and it is the interpretation of the arguments $\left\{\boldsymbol{\ell}_{a}\right\}$ of the energy density as generators of a discrete subgroup corresponding to dislocation density tensor $S$ that forces us to solve that problem. (If the arguments $\left\{\boldsymbol{\ell}_{a}\right\}$ were to be interpreted as elements of the corresponding Lie algebra, then the dependence of $w$ on these elements would have to recognize that, generally, the relevant exponential function is not surjective). Note that, according to (5.19), the correspondence between $S$ and the quantity $\theta$ is generally infinitely many to one - so if the energy density is to depend on the 'local structure' (i.e. on the discrete subgroup $\mathcal{G}$ that is determined by $\theta$, one must require that

$$
w\left(\left\{\boldsymbol{\ell}_{a}\right\}, S\right)=\tilde{w}\left(\left\{\boldsymbol{\ell}_{a}\right\}, \theta\right),
$$

for some constitutive function $\tilde{w}$. Furthermore, if $\left\{\boldsymbol{\ell}_{a}\right\}$ is a set of generators of the same discrete subgroup (satisfying conditions (7.6), (8.4), (8.5)) then

$$
\tilde{w}\left(\left\{\boldsymbol{\ell}_{a}\right\}, \theta\right)=\tilde{w}\left(\left\{\boldsymbol{\ell}_{a}^{\prime}\right\}, \theta\right)
$$


Now changes of generators induce mappings of group elements (expressed as products of the generators and their inverses) in the obvious way. Only some changes of generators preserve the group structure (according to Magnus, Karrass and Solitar [15], they are the changes that preserve the commutator relations that define the group, in this case) - they are automorphisms of the discrete subgroup. For some of the groups that we have considered here and in [11], it is a fact that those automorphisms (of the discrete subgroup) extend to automorphisms of the ambient Lie group, i.e., they are restrictions (to the discrete subgroup) of elastic deformations of the continuum (viewed as a Lie group). Therefore we can classify the groups, and corresponding changes of generators as follows:

(i) Discrete groups whose automorphisms extend to automorphisms of the ambient Lie group, and those where this is not so (it seems that there is no 'intermediate' category);

(ii) (a) Changes of generators which provide automorphisms of the discrete subgroup, where these are restrictions of elastic deformations;

(b) Changes of generators which provide automorphisms of the discrete subgroup which are not restrictions of elastic deformations;

(c) Change of generators which do not provide automorphisms of the discrete subgroup.

In other words, the symmetries may be classified according as to whether or not they are 'elastic' or 'inelastic', and furthermore the inelastic symmetries may be categorized according to whether or not they preserve the discrete group structure or not (in the sense that the commutator relations that define the group are preserved or not).

We hope to investigate these issues in detail in future work, and provide an explicit classification types of symmetry, for each class of defective crystals. We also intend to correlate those findings with the observed 'slip systems' in different classes of solid crystals.

Finally, we have in the past accepted the view that the energy density depends just on the 'points' of $\mathbb{R}^{3}$ generated in the iteration procedure of section 4 . The argument is that if a certain choice of generators and corresponding group (determined by $\left\{\boldsymbol{\ell}_{a}\right\}, S$ ) gives a certain discrete group, and hence a set of points in $\mathbb{R}^{3}$, then the energy density is unchanged if a different choice of generators and corresponding group (determined by $\left\{\ell_{a}^{\prime}\right\}, S^{\prime}$ ) leads to the set of points in 
$\mathbb{R}^{3}$. (One may view the energy density as the local potential energy per unit volume of this set of points, with the interaction energy between pairs, triplets, etc., of points determined by the relevant Euclidean distances.) This argument disregards the following fact, that not all elements, pairs of group elements, etc., in the discrete group constructed from $\left\{\boldsymbol{\ell}_{a}\right\}, S$ are equivalent, e.g, if $\boldsymbol{g}, \boldsymbol{h} \in \mathcal{G}$, then either $\boldsymbol{g} \boldsymbol{h}^{-1} \in \mathcal{G}^{\prime}$ or $\boldsymbol{g} \boldsymbol{h}^{-1} \notin \mathcal{G}^{\prime}$. (Said differently, given $\boldsymbol{g} \in \mathcal{G}$, only some group elements represent the final point of a Burger's path with starting point $\boldsymbol{g}$ ). In truth, the choice of energy density is just a constitutive assumption, but if one accepts that the Lie group structure determined by the quantities $\left\{\boldsymbol{\ell}_{a}\right\}, S$ determines the symmetries of $w$, then it is sensible to require (at the least) that the symmetries are determined not only by the set of points in $\mathbb{R}^{3}$ determined by $\mathcal{G}$, but also the set of points in $\mathbb{R}^{3}$ determined by $\mathcal{G}^{\prime}$. That is the point of view that has been taken in this paper, for we have determined the allowed changes of generators by first of all restricting attention to $\mathcal{G}^{\prime}$, rather than $\mathcal{G}$. (Note that $\theta$, in $(9.1)$, determines $\mathcal{G}^{\prime}$ as well as $\mathcal{G}$ ). Therefore the view taken here is less restrictive that the view taken in the past, in particular in [11]. We hope that our forthcoming attempt to correlate these symmetries with observed slip systems in crystals will provide some evidence with which to assess the assumptions that have been made.

\section{Acknowledgement}

We acknowledge the support of the UK Engineering and Physical Sciences Research Council through grant EP/G047162/1.

\section{References}

[1] P. J. Olver, Equivalence, invariants, and symmetry. Cambridge University Press, Cambridge 1996.

[2] Davini, C., A proposal for a continuum theory of defective crystals, Archive for Rational Mechanics and Analysis, 96, 295-317 (1986).

[3] Davini, C. and Parry, G. P., On defect preserving deformations in crystals, International Journal of Plasticity 5, 337-369 (1989).

[4] G. P. Parry and M. Šilhavý, Elastic scalar invariants in the theory of defectivve crystals, Proc. R. Soc. Lond. A, 455, 1999, pp. 4333-4346.

[5] L.S. Pontryagin, Topological Groups (2nd Edition) Gordon and Breach, New York, London, Paris, 1955. 
[6] Auslander, L., Green, L. and Hahn, F., Flows on homogeneous spaces, Annals of Mathematics Studies, Number 53, Princeton University Press, Princeton, New Jersey, 1963.

[7] Elzanowski, M. and Parry, G. P., Material symmetry in a theory of continuously defective crystals, Journal of Elasticity, 74, 215-237 (2004).

[8] Jacobson, N., Lie Algebras, Dover Publicatins, Inc. New York, 1979.

[9] Cermelli, P. and Parry, G. P., The structure of uniform discrete defective crystals, Continuum Mechanics and Thermodynamics, 18, 47-61 (2006).

[10] Parry, G. P. and Sigrist, R., Reconciliation of local and global symmetries for a class of crystals with defects, Journal of Elasticity, OnlineFirst, 4 May 2011 (doi:10.1007/s10659011-9342-5).

[11] Nicks, R. and Parry, G. P. On symmetries of crystals with defects related to a class of solvable groups, Part I: $S_{1}$.

[12] Rossmann, W., Lie groups. An introduction through Linear Groups, Oxford University Press, Oxford, New York, 2002.

[13] Conrad, K., Decomposing $S L_{2}(\mathbb{R}), \quad$ http://www.math.uconn.edu/ kcon$\mathrm{rad} /$ blurbs/grouptheory/SL(2,R).pdf

[14] Bachmuth, S., Automorphisms of free metabelian groups, Transactions of the American Mathematical Society, 118, 93-104 (1965).

[15] Magnus, W., Karrass, A. and Solitar, D., Combinatorial Group Theory, Dover, New York, 1976.

[16] Thurston, W., Three dimensional geometry and topology, vol. 1, Princeton University Press, Princeton, New Jersey, 1997. 